\title{
NEW DETECTIONS OF HNC IN PLANETARY NEBULAE: EVOLUTION OF THE $[\mathrm{HCN}] /[\mathrm{HNC}]$ RATIO
}

\author{
D. R. SCHMIDT ${ }^{1,2}$ AND L. M. ZiURYs ${ }^{1,2,3}$ \\ ${ }^{1}$ Department of Astronomy and Steward Observatory, University of Arizona, 933 N. Cherry Avenue Tucson, AZ 85721-0065, USA; drschmidt@email.arizona.edu \\ 2 Arizona Radio Observatory, University of Arizona, 933 N. Cherry Avenue Tucson, AZ 85721-0065, USA \\ ${ }^{3}$ Department of Chemistry and Biochemistry, University of Arizona, 933 N. Cherry Avenue Tucson, AZ 85721-0065, USA \\ Received 2016 September 26; revised 2016 November 10; accepted 2016 November 18; published 2017 January 19
}

\begin{abstract}
New detections of HNC have been made toward 11 planetary nebulae (PNe), including K4-47, K3-58, K3-17, M328, and M4-14. These sources, which represent a wide range of ages and morphologies, had previously been observed in HCN by Schmidt \& Ziurys. Measurements of the $J=1 \rightarrow 0$ and $J=3 \rightarrow 2$ transitions of HNC near 90 and $271 \mathrm{GHz}$ were conducted using the new $12 \mathrm{~m}$ and the Sub-Millimeter Telescope of the Arizona Radio Observatory. $\mathrm{HCN}$ and $\mathrm{HNC}$ were also identified via their $J=1 \rightarrow 0$ lines toward eight positions across the Helix Nebula (NGC 7293). Column densities for HNC, determined from radiative transfer modeling, were $N_{\text {tot }}\left(\right.$ HNC) $\sim(0.06-4.0) \times 10^{13} \mathrm{~cm}^{-2}$, corresponding to fractional abundances with respect to $\mathrm{H}_{2}$ of $f$ $\sim(0.02-1.4) \times 10^{-7}$. The $\mathrm{HCN}$ and HNC column densities across the Helix were found to be $N_{\text {tot }}(\mathrm{HCN}) \sim(0.2-2.4) \times 10^{12} \mathrm{~cm}^{-2}$ and $N_{\text {tot }}(\mathrm{HNC}) \sim(0.07-1.6) \times 10^{12} \mathrm{~cm}^{-2}$, with fractional abundances of $(0.2-3.2) \times 10^{-7}$ and $(0.09-2.2) \times 10^{-7}$. The $[\mathrm{HCN}] /[\mathrm{HNC}]$ ratio varied between $\sim 1-8$ for all $\mathrm{PNe}$, with $[\mathrm{HCN}] /[\mathrm{HNC}] \sim 1-4$ across the Helix. These values are greatly reduced from what has been found in asymptotic giant branch stars, where the ratio is typically $>100$. Both the abundance of $\mathrm{HNC}$ and the $[\mathrm{HCN}] /[\mathrm{HNC}]$ ratio do not appear to vary significantly with nebular age across a time span of $\sim 10,000$ years, in contrast to predictions of chemical models. The increase in HNC appears to arise in the proto-planetary stage, but becomes "frozen" once the PN phase is reached.
\end{abstract}

Key words: astrochemistry - ISM: molecules - planetary nebulae: general - radio lines: ISM

\section{INTRODUCTION}

Planetary nebulae (PNe) form from intermediate mass $\left(\sim 0.5-8 M_{\text {sol }}\right)$ stars as they leave the asymptotic giant branch (AGB). While in the AGB phase, these stars experience extensive mass loss, sometimes as high as $\sim 10^{-4} M_{\mathrm{sol}} \mathrm{yr}^{-1}$. Atomic and molecular material is stripped from the surface of the star in the form of a superwind, leading to the creation of a circumstellar envelope (e.g., Kwok 2000). Once the stellar core, known as a white dwarf, reaches a temperature of $\sim 30,000 \mathrm{~K}$, mass loss ceases and the core is hot enough to ionize the surrounding remnant shell material, resulting in a PN.

The PN central white dwarf generates a significant ultraviolet (UV) radiation field during its transition from the proto-PN stage to the PN stage, typically $\sim 10^{5}$ times that of the general interstellar medium (ISM), but decreasing to $10-100$ as the remnant envelope expands and the luminosity of the white dwarf declines (Cox 1997). The copious amount of UV photons penetrating the nebula is predicted to photodissociate the remnant molecular material from the AGB stage within a thousand years (e.g., Redman et al. 2003). Indeed, the first molecular searches of PNe turned up negative (e.g., Penzias et al. 1971; Wilson et al. 1974). Subsequently, both $\mathrm{CO}$ and $\mathrm{H}_{2}$ have been detected in numerous PNe (e.g., Huggins \& Healy 1989; Huggins et al. 1996, 2005; Hora et al. 1999; Likkel et al. 2006). More recent studies have identified other molecules in planetary nebulae with ever increasing complexity. Toward the young PNe NGC 7027 and NGC 6537, for example, $\mathrm{HCN}, \mathrm{HNC}, \mathrm{CCH}, \mathrm{CS}, \mathrm{SO}, \mathrm{H}_{2} \mathrm{CO}, \mathrm{HCO}^{+}$, and $\mathrm{N}_{2} \mathrm{H}^{+}$ have been identified (Zhang et al. 2008; Edwards \& Ziurys 2013). In more evolved nebulae, species such as $\mathrm{HCN}, \mathrm{HNC}, \mathrm{HCO}^{+}$, CS, and $\mathrm{CN}$ have been found (e.g., Cox et al. 1992; Bachiller et al. 1997; Edwards et al. 2014). The very old Helix Nebula (age $\sim 12,000$ years.) has an assortment of interesting molecules, including $\mathrm{HCO}^{+}, \mathrm{CN}, \mathrm{HCN}, \mathrm{H}_{2} \mathrm{CO}$, $\mathrm{CCH}, \mathrm{c}-\mathrm{C}_{3} \mathrm{H}_{2}$, and $\mathrm{HNC}$ (Bachiller et al. 1997; Tenenbaum et al. 2009), and mapping data have shown that the molecular material is distributed throughout the PN (Zack \& Ziurys 2013; Zeigler et al. 2013). SiO, SO, and $\mathrm{SO}_{2}$ have recently been found in M2-48 (age $\sim 5000$ years.) as well (Edwards \& Ziurys 2014). Perhaps even more striking is the identification of $\mathrm{C}_{60}$ in several PNe with low central star temperatures (Cami et al. 2010; García-Hernández et al. 2010, 2011, 2012; Otsuka et al. 2013). The presence of long-lived molecular material is believed to arise from shielding by high-density clumps (Howe et al. 1994), as seen in images of the Helix and the Ring (e.g., Meaburn et al. 1992; Speck et al. 2003; Meixner et al. 2005).

Very recently, Schmidt \& Ziurys (2016) conducted a search for $\mathrm{HCN}$ and $\mathrm{HCO}^{+}$in a set of 17 nebulae that had previously only been detected in $\mathrm{CO}$. The target sources spanned a range of ages, angular sizes, and morphologies. Of the 17 target objects, 13 were detected in either $\mathrm{HCN}$ or $\mathrm{HCO}^{+}$, with both molecules common to most sources. $\mathrm{HCN}$ and $\mathrm{HCO}^{+}$ abundances, relative to $\mathrm{H}_{2}$, were found to be in the range $\sim(0.1-9.1) \times 10^{-7}$ and $\sim(0.04-7.4) \times 10^{-7}$, respectively (Schmidt \& Ziurys 2016).

With this new target list in hand, we undertook a search for $\mathrm{HNC}$, the metastable isomer of hydrogen cyanide, with the goal of measuring $[\mathrm{HCN}] /[\mathrm{HNC}]$ ratios. Models predict that this ratio should be near unity in PNe (e.g., Kimura et al. 2012), because the formation mechanism of both molecules is thought to occur through the dissociative recombination of $\mathrm{HCNH}^{+}$:

$$
\mathrm{HCNH}^{+}+e^{-} \rightarrow \mathrm{HNC}, \mathrm{HCN}+\mathrm{H}
$$

Theory indicates that this process produces HCN and HNC in equal amounts (Talbi 1999). Temperature also influences the ratio; values as high as $\sim 80$ are observed toward the warm, 
Table 1

Transition Frequencies and Telescope Parameters

\begin{tabular}{lccccc}
\hline \hline Molecule & Transition & Frequency (MHz) & Telescope & $\theta_{\mathrm{B}}\left({ }^{\prime \prime}\right)$ & $\eta_{\mathrm{B}}$ \\
\hline HNC & $J=1 \rightarrow 0$ & $90,663.6$ & $12 \mathrm{~m}$ & 69 & 0.88 \\
& $J=3 \rightarrow 2$ & $271,981.1$ & SMT & 28 & 0.75 \\
HCN & $J=1 \rightarrow 0$ & $88,631.8$ & $12 \mathrm{~m}$ & 71 & 0.88 \\
\hline
\end{tabular}

star-forming region Orion KL (Schilke et al. 1992), but drop to near unity in the cold gas in TMC-1 (e.g., Irvine \& Schloerb 1984). In AGB stars, the ratio is far from unity, because hot, LTE chemistry in the inner envelope favors the more stable $\mathrm{HCN}$. HNC is created in the outer envelope from $\mathrm{HCNH}^{+}$, a product of cosmic-ray ion-molecule chemistry (e.g., Daniel et al. 2012). The $[\mathrm{HCN}] /[\mathrm{HNC}]$ ratio is thought to decrease from the AGB into the protoplanetary nebula and PN stages. HNC and HCN have been detected in a very limited number of $\mathrm{PNe}$, however, and therefore accurate values of the $[\mathrm{HCN}] /[\mathrm{HNC}]$ ratio in these objects are rare. It is thus unclear how this ratio evolves over the lifetime of a PN.

Here we report the results of our search for $\mathrm{HNC}$ in PNe. A set of 11 nebulae were studied in the $J=1 \rightarrow 0$ and $3 \rightarrow 2$ transitions of this molecule using the facilities of the Arizona Radio Observatory (ARO). We also present measurements of both $\mathrm{HCN}$ and HNC at eight new positions in the Helix Nebula. In this paper we describe the details of our observations, results, and analysis, and examine the evolution of the $[\mathrm{HCN}] /[\mathrm{HNC}]$ ratio with nebular age.

\section{OBSERVATIONS}

Measurements of the $J=1 \rightarrow 0$ and $J=3 \rightarrow 2$ transitions of HNC, as well as the $J=1 \rightarrow 0$ transition of $\mathrm{HCN}$ in the Helix, were performed between 2013 February and 2016 June using the facilities of the ARO. Details of the observations, including line frequencies, beam sizes, and beam efficiencies, can be found in Table 1 . The $3 \mathrm{~mm}$ measurements $(J=1 \rightarrow 0$ transitions of $\mathrm{HNC}$ and $\mathrm{HCN}$ ) were conducted with the new $12 \mathrm{~m}$ "European" ALMA prototype antenna at Kitt Peak. The dual-polarization receiver on the $12 \mathrm{~m}$ consists of ALMA Band 3 sideband-separating (SBS) mixers. Data were obtained in the lower sideband, with the image rejection, intrinsic in the mixer architecture, typically greater than $15 \mathrm{~dB}$. The temperature scale, $T_{A}^{*}$, was determined by the chopper wheel method, and is related to the main beam brightness temperature, $T_{\mathrm{R}}$, by the equation $T_{\mathrm{R}}=T_{A}^{*} / \eta_{\mathrm{b}}$, where $\eta_{\mathrm{b}}$ is the main beam efficiency. The estimated calibration accuracy of the temperature scale is $\sim 20 \%$. Three 256-channel filter banks with $1 \mathrm{MHz}, 250 \mathrm{kHz}$, and $500 \mathrm{kHz}$ resolutions, respectively, and the Millimeter Autocorrelator (MAC), were employed as backends. The MAC has 2048 channels with $781.2 \mathrm{kHz}$ spectral resolution. All backends were operated in parallel mode.

Observations of the $J=3 \rightarrow 2$ transition of HNC were made using the $1.3 \mathrm{~mm}$ receiver at the ARO $10 \mathrm{~m}$ SubMillimeter Telescope (SMT) on Mt. Graham, AZ. This receiver employs ALMA Band 6 SBS mixers. These observations were performed in upper sideband (USB), with average image rejections of $25 \mathrm{~dB}$ and $16 \mathrm{~dB}$ in horizontal and vertical polarizations, respectively. The temperature scale was determined using the chopper wheel method, and is given as $T_{A}^{*}$. These observations had an estimated calibration accuracy of $20 \%$. Two filter banks were employed as backends, both operated in parallel mode: one with 2048 channels and $1 \mathrm{MHz}$ resolution, and the other with 512 channels and $250 \mathrm{kHz}$ resolution.

Summaries of the sources, including estimates for ages and sizes, are provided in Table 2. An in-depth discussion of nebular ages, how they were derived, and their uncertainties is given in Schmidt \& Ziurys (2016). The average age uncertainty is $\sim 30 \%$. Positions observed in the Helix were those used by Zack \& Ziurys (2013); see their Table 1 (offsets are from the central star in R.A. and decl.). Observations were conducted in either position-switching or beam-switching mode, as determined by the source size. For most objects for which position switching was required, an offset of $+30^{\prime}$ in azimuth was used. In the case of $\mathrm{Hb} 5$, an offset of $-30^{\prime}$ in azimuth was utilized because of Galactic contamination. Beam-switching was performed using a throw of $\pm 2^{\prime}$. Local oscillator shifts were made to test for image contamination, and pointing was checked hourly using nearby strong continuum sources (ideally Jupiter or Mars when available). The average rms for these observations was $\sim 3 \mathrm{mK}$.

\section{RESULTS}

HNC was detected in all of the 11 sources studied, typically in both transitions. Figures 1(a)-(d) display the spectra, while Table 3 summarizes the corresponding measured line parameters: peak antenna temperatures $T_{A}^{*}$, LSR velocities, and FWHM line widths, determined by Gaussian or shell fits to the line profiles. Note that $\mathrm{HNC}$ has no appreciable hyperfine structure in either transition studied. Observed line intensities for $\mathrm{HNC}$ were in the range $\sim 2-12 \mathrm{mK}$. LSR velocities and line widths were strikingly similar to those found for $\mathrm{HCN}$ in these sources, as highlighted in Figure 1(a) for K4-47 (also c.f. Schmidt \& Ziurys 2016).

The $J=1 \rightarrow 0$ transitions of both $\mathrm{HCN}$ and $\mathrm{HNC}$ were detected at all eight positions in the Helix. Bachiller et al. (1997) had detected both molecules at the $\left(-372^{\prime \prime}, 0^{\prime \prime}\right)$ position, which was not included in our set. Figure 2 displays the spectra as a function of position in the Helix. The $\left(130^{\prime \prime},-180^{\prime \prime}\right)$ position shows the $\mathrm{HCN}$ hyperfine pattern underneath one feature. As the figure illustrates, multiple velocity components are present at nearly every position, and the three quadrupole hyperfine lines of $\mathrm{HCN}$ are often visible. The line profiles also closely resemble those measured in $\mathrm{CO}, \mathrm{HCO}^{+}$, and $\mathrm{H}_{2} \mathrm{CO}$ by Zack \& Ziurys (2013).

Table 4 lists the line intensities $\left(T_{A}^{*}\right)$, LSR velocities, and line widths for each velocity component and the individual hyperfine lines of $\mathrm{HCN}$, when visible, for the Helix. The peak temperatures ranged between $\sim 5-35 \mathrm{mK}$ for the main hyperfine line $(F=2 \rightarrow 1)$ of $\mathrm{HCN}$ and $\sim 8-60 \mathrm{mK}$ for HNC. These parameters were determined from Gaussian fits to the line profiles, and assuming an LTE hyperfine intensity ratio of $3: 5: 1 \quad(F=1 \rightarrow 1: 2 \rightarrow 1: 1 \rightarrow 0)$ for the $\mathrm{HCN}$ data-a reasonable assumption on examining the line profiles. Often the hyperfine and velocity components were intermixed for this molecule, but the HNC line profiles provided a good template for the velocity structure at each position, as did previous data (Zack \& Ziurys 2013). Examples of the fitted HCN line profiles are shown in Figure 3. Here, spectra with overlaid fits (in green or grayscale) from the $\left(-15^{\prime \prime}, 270^{\prime \prime}\right)$ and $\left(-435^{\prime \prime}, 75^{\prime \prime}\right)$ positions are shown. At first glance, the $\left(-15^{\prime \prime}, 270^{\prime \prime}\right)$ data give the impression of non-LTE hyperfine intensities in HCN, but this is actually the result of two closely spaced velocity components 
Table 2

Target List of Planetary Nebulae

\begin{tabular}{|c|c|c|c|c|c|c|}
\hline Source & $\alpha(\mathrm{J} 2000)(\mathrm{h} \mathrm{m} \mathrm{s})$ & $\delta(\mathrm{J} 2000)\left({ }^{\circ} \prime \prime \prime\right)$ & $\theta_{\mathrm{S}}\left({ }^{\prime \prime}\right)$ & Age (years) & Morphology & $\mathrm{C} / \mathrm{O}^{\circ}$ \\
\hline $\mathrm{Hb} 5$ & $17: 47: 56.2$ & $-29: 59: 41.9$ & $31^{\mathrm{a}}$ & $1500^{\mathrm{b}}$ & bipolar & C-rich (?) \\
\hline K3-17 & $18: 56: 18.2$ & $+07: 07: 25.9$ & $8^{\mathrm{c}}$ & $970^{\mathrm{d}}$ & bipolar & C-rich (?) \\
\hline $\mathrm{K} 3-45$ & $19: 46: 15.6$ & $+24: 11: 03.7$ & $7^{\mathrm{e}}$ & $830^{f}$ & bipolar & $\ldots$ \\
\hline $\mathrm{K} 3-58$ & $20: 21: 58.3$ & $+29: 59: 21.1$ & $17^{\mathrm{g}}$ & $11070^{\mathrm{g}}$ & bipolar & $\ldots$ \\
\hline M1-7 & $06: 37: 21.0$ & $+24: 00: 36.6$ & $11^{\mathrm{c}}$ & $6000^{c}$ & bipolar & $1.1^{\mathrm{h}}$ \\
\hline M3-28 & $18: 32: 41.3$ & $-10: 05: 50.0$ & $17^{\mathrm{a}}$ & $7700^{c}$ & quadrupolar & $\ldots$ \\
\hline M3-55 & $18: 33: 14.8$ & $-10: 15: 19.5$ & $10^{\mathrm{g}}$ & $5040^{\mathrm{g}}$ & bipolar & $\ldots$ \\
\hline M4-14 & $19: 21: 00.7$ & $+07: 36: 52.4$ & $15^{\mathrm{g}}$ & $5550^{\mathrm{g}}$ & quadrupolar & $\ldots$ \\
\hline NGC 2440 & $07: 41: 54.9$ & $-18: 12: 29.7$ & $38^{\mathrm{a}}$ & $2960^{\mathrm{i}}$ & quadrupolar (?) & C-rich ${ }^{j}$ \\
\hline NGC $6772 a^{m}$ & $19: 14: 37.3$ & $-02: 42: 19.8$ & $76^{\mathrm{a}}$ & $10900^{\mathrm{k}}$ & elliptical & $\ldots$ \\
\hline NGC $6772 b^{n}$ & $19: 14: 35.4$ & $-02: 42: 25.0$ & $\ldots$ & $\ldots$ & $\cdots$ & $\ldots$ \\
\hline K4-47 & $04: 20: 45.2$ & $+56: 18: 12.1$ & $8^{c}$ & $900^{1}$ & bipolar & C-rich (?) \\
\hline
\end{tabular}

Notes.

a Tylenda et al. (2003).

b López et al. (2012).

c Phillips \& Márquez-Lugo (2011).

d Based on distance from Phillips \& Márquez-Lugo (2011).

e Acker et al. (1992).

f Giammanco et al. (2011).

g Dobrinčić et al. (2008).

h Patriarchi \& Perinotto (1994).

${ }^{\mathrm{i}}$ Górny et al. (1997).

j Pottasch \& Bernard-Salas (2010), Dufour et al. (2015).

k Ali et al. (2012).

${ }^{1}$ Corradi et al. (2000).

m Milne (1973), converted to J2000.0 coordinates.

${ }^{n}$ Kerber et al. (2003).

${ }^{\circ}$ see Schmidt \& Ziurys (2016).

(see Table 4$)$. The complexity in the $\left(-435^{\prime \prime},+75^{\prime \prime}\right)$ spectrum is a product of three velocity features.

\subsection{Survey Sources}

$H b$ 5: The $J=1 \rightarrow 0$ transition of HNC was detected with a central velocity of $-4.6 \mathrm{~km} \mathrm{~s}^{-1}$ in this $\mathrm{PN}$, close to the systemic velocity of the star, which is $-7 \mathrm{~km} \mathrm{~s}^{-1}$ (López et al. 2012). As for $\mathrm{HCN}$ and $\mathrm{HCO}^{+}$, no obvious Galactic contamination was present in the spectrum. The line profile appears asymmetric, and the line width of $\sim 33 \mathrm{~km} \mathrm{~s}^{-1}$ is comparable to that of the $J=3 \rightarrow 2$ transition of $\mathrm{HCN}$ $\left(\sim 40 \mathrm{~km} \mathrm{~s}^{-1}\right)$. López et al. (2012) calculated a kinematic age of 1500 years for this source, based on the radial expansion velocity.

K3-17: HNC was detected in the $J=3 \rightarrow 2$ transition of this source, with $V_{\mathrm{LSR}} \sim 32 \mathrm{~km} \mathrm{~s}^{-1}$ and $\Delta V_{1 / 2} \sim 50 \mathrm{~km} \mathrm{~s}^{-1}$, consistent with the $J=3 \rightarrow 2$ lines of both $\mathrm{HCN}$ and $\mathrm{HCO}^{+}$. The nebula is small $\left(8^{\prime \prime}\right)$ and multi-polar with several secondary loops. Multiple narrow velocity components at $\sim 20,30,39$, and $57 \mathrm{~km} \mathrm{~s}^{-1}$ appear to be visible in the spectra of HNC, $\mathrm{HCN}$, and $\mathrm{HCO}^{+}$, indicative of this complex geometry. An age of $\sim 1000$ years was derived for K3-17 from the expansion velocity inferred from CO (Schmidt \& Ziurys 2016).

K3-45: Only the $J=3 \rightarrow 2$ transition of HNC was detected in this small (6!"8: Acker et al. 1992) bipolar object. The observed $V_{\mathrm{LSR}}$ of $\sim 34 \mathrm{~km} \mathrm{~s}^{-1}$ and $\Delta V_{1 / 2}$ of $\sim 34 \mathrm{~km} \mathrm{~s}^{-1}$ are comparable to those seen in $\mathrm{HCN}$. The HNC line profile is clearly double-peaked, with maxima at $\sim 18 \mathrm{~km} \mathrm{~s}^{-1}$ and $\sim 45 \mathrm{~km} \mathrm{~s}^{-1}$, as also seen in the HCN $J=3 \rightarrow 2$ transition. Schmidt \& Ziurys (2016) calculated an age estimate of $\sim 800$ years for this nebula.
$K 3-58$ : Both the $J=1 \rightarrow 0$ and $3 \rightarrow 2$ transitions of HNC were observed in this small $\left(17^{\prime \prime}\right)$ bipolar nebula. The LSR velocities of $\sim 20 \mathrm{~km} \mathrm{~s}^{-1}$ and line widths of $\sim 30 \mathrm{~km} \mathrm{~s}^{-1}$ are consistent with the $\mathrm{HCN}$ and $\mathrm{HCO}^{+}$spectra. An age of $\sim 11,000$ years was estimated for this source by Dobrinčić et al. (2008) from their spatio-kinematical study.

$M 1-7:$ The $J=1 \rightarrow 0$ and $3 \rightarrow 2$ transitions of $\mathrm{HNC}$ were both detected in this small, older, compact $\left(\sim 11^{\prime \prime}\right)$ bipolar PN. The line widths and LSR velocities are consistent with what is seen for $\mathrm{HCN}$ and $\mathrm{HCO}^{+}$within the uncertainties. There may be additional velocity structure in the spectra near $-29 \mathrm{~km} \mathrm{~s}^{-1}$ and $-7 \mathrm{~km} \mathrm{~s}^{-1}$. Schmidt \& Ziurys (2016) derived an age of $\sim 6000$ years for M1-7.

M3-28: HNC was detected in both transitions in this small $\left(\sim 17^{\prime \prime}\right)$ quadrupolar nebula. As was the case for both HCN and $\mathrm{HCO}^{+}$, the $J=1 \rightarrow 0$ transition of $\mathrm{HNC}$ was contaminated by Galactic emission toward redshifted velocities. Thus, the $V_{\mathrm{LSR}}$ and $\Delta V_{1 / 2}$ of the $J=1 \rightarrow 0$ transition $\left(\sim 33 \mathrm{~km} \mathrm{~s}^{-1}\right.$ and $\sim 35 \mathrm{~km} \mathrm{~s}^{-1}$ respectively) are estimates. The line parameters of the $J=3 \rightarrow 2$ transition match reasonably well with those observed for the $\mathrm{HCN}$ and $\mathrm{HCO}^{+}$transitions $\left(V_{\mathrm{LSR}} \sim 33 \mathrm{~km} \mathrm{~s}^{-1}\right.$ and $\left.\Delta V_{1 / 2} \sim 36-42 \mathrm{~km} \mathrm{~s}^{-1}\right)$. An adopted age of $\sim 7700$ years was obtained by Schmidt \& Ziurys (2016).

M3-55: While both transitions were detected in this small ( 9". 5) bipolar PN, the $J=1 \rightarrow 0$ spectrum was partially obscured by Galactic contamination, making estimates of the line parameters less reliable. The $V_{\mathrm{LSR}}$ and $\Delta V_{1 / 2}$ of the $J=3 \rightarrow 2$ transition, $\sim 30 \mathrm{~km} \mathrm{~s}^{-1}$ and $\sim 23 \mathrm{~km} \mathrm{~s}^{-1}$ respectively, are comparable to those seen for the $J=3 \rightarrow 2$ transition of both $\mathrm{HCN}$ and $\mathrm{HCO}^{+}$. An age of $\sim 5000$ years is given by Dobrinčić et al. (2008). 

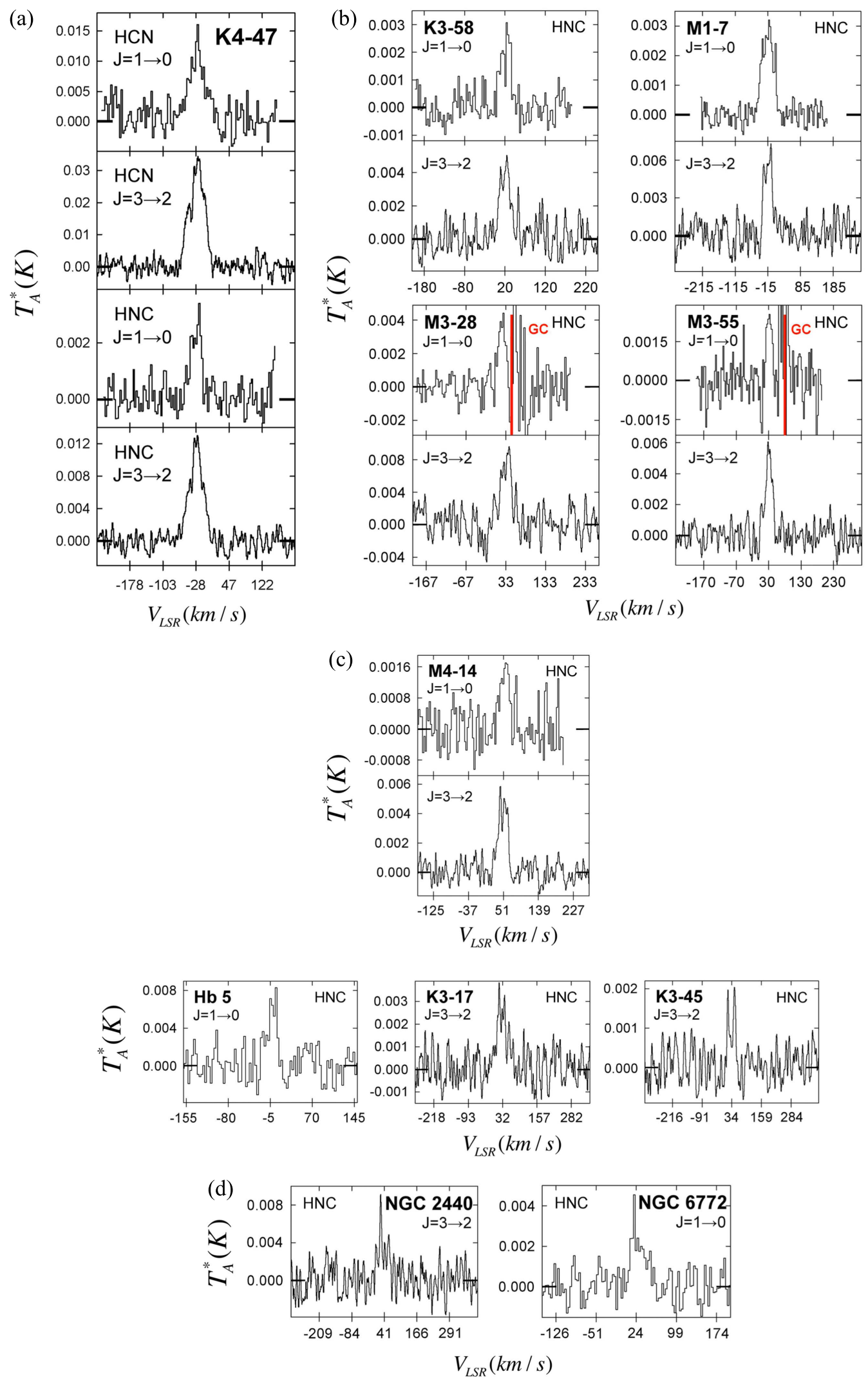

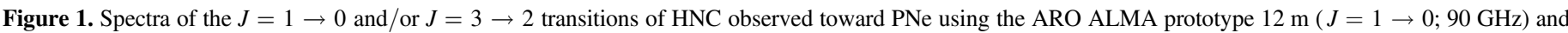

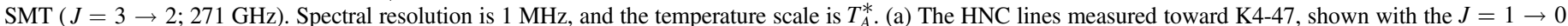

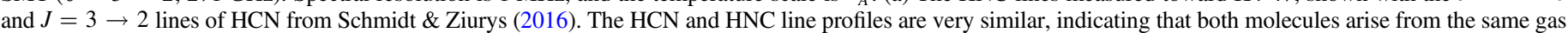

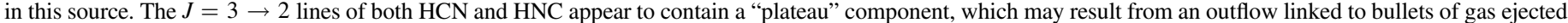

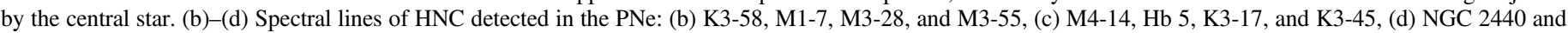

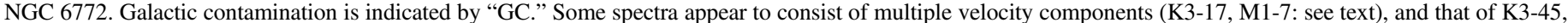
a bipolar nebula, is clearly double-peaked. 
Table 3

Observations of HNC: $J=1 \rightarrow 0$ and $J=3 \rightarrow 2$ Toward Planetary Nebula

\begin{tabular}{llccc}
\hline \hline Name & Transition & $T_{A}^{*}(\mathrm{mK})$ & $V_{\text {LSR }}\left(\mathrm{km} \mathrm{s}^{-1}\right)$ & $\Delta V_{1 / 2}\left(\mathrm{~km} \mathrm{~s}^{-1}\right)$ \\
\hline Hb 5 & $J=1 \rightarrow 0$ & $6.9 \pm 1.2$ & $-4.6 \pm 3.3$ & $33.1 \pm 6.6$ \\
& $J=3 \rightarrow 2$ & $<5$ & $\ldots$ & $\ldots$ \\
K3-17 & $J=1 \rightarrow 0$ & $<1$ & $\ldots$ & $\ldots$ \\
& $J=3 \rightarrow 2$ & $2.6 \pm 1.1$ & $32.0 \pm 4.4$ & $49.9 \pm 6.6$ \\
K3-45 & $J=1 \rightarrow 0$ & $<2$ & $\ldots$ & $\ldots$ \\
& $J=3 \rightarrow 2^{\mathrm{a}}$ & $1.8 \pm 0.5$ & $33.9 \pm 2.2$ & $34.2 \pm 2.2$ \\
K3-58 & $J=1 \rightarrow 0$ & $2.8 \pm 0.3$ & $20.7 \pm 3.3$ & $30.5 \pm 6.6$ \\
& $J=3 \rightarrow 2$ & $4.0 \pm 1.3$ & $20.2 \pm 2.2$ & $33.8 \pm 3.3$ \\
M1-7 & $J=1 \rightarrow 0$ & $2.8 \pm 0.9$ & $-16.6 \pm 6.6$ & $42.2 \pm 6.6$ \\
& $J=3 \rightarrow 2$ & $6.0 \pm 2.0$ & $-13.6 \pm 2.2$ & $31.2 \pm 4.4$ \\
M3-28 & $J=1 \rightarrow 0^{\mathrm{b}}$ & $4.6 \pm 0.7$ & $\sim 33$ & $\sim 35$ \\
& $J=3 \rightarrow 2$ & $8.5 \pm 1.5$ & $33.5 \pm 2.2$ & $36.0 \pm 3.3$ \\
M3-55 & $J=1 \rightarrow 0^{\mathrm{b}}$ & $2.7 \pm 0.4$ & $29.2 \pm 3.3$ & $20.3 \pm 3.3$ \\
& $J=3 \rightarrow 2$ & $5.6 \pm 1.0$ & $30.3 \pm 2.2$ & $23.1 \pm 2.2$ \\
M4-14 & $J=1 \rightarrow 0$ & $1.6 \pm 0.5$ & $52.9 \pm 3.3$ & $26.9 \pm 3.3$ \\
& $J=3 \rightarrow 2$ & $5.0 \pm 1.1$ & $48.8 \pm 2.2$ & $26.1 \pm 2.2$ \\
NGC 2440 & $J=1 \rightarrow 0$ & $<1$ & $\ldots$ & $\ldots$ \\
& $J=3 \rightarrow 2$ & $\sim 7$ & $40.7 \pm 4.4$ & $\sim 50$ \\
NGC 6772 & $J=1 \rightarrow 0$ & $2.7 \pm 1.2$ & $24.2 \pm 6.6$ & $31.3 \pm 6.6$ \\
& $J=3 \rightarrow 2$ & $<10$ & $\ldots$ & $\ldots$ \\
NGC 6772 & & \\
& $J=3 \rightarrow 2$ & $<7$ & $\ldots$ & $\ldots$ \\
K4-47 & $J=1 \rightarrow 0$ & $2.9 \pm 1.0$ & $-28.4 \pm 3.3$ & $29.8 \pm 3.3$ \\
& $J=3 \rightarrow 2$ & & $11.5 \pm 2.0$ & \\
& & & & $-27.8 \pm 2.2$
\end{tabular}

$36.9 \pm 3.3$

Notes.

${ }^{\mathrm{a}}$ Fit with shell profile.

${ }^{\mathrm{b}}$ Strong galactic contamination.

M4-14: In this $15^{\prime \prime}$ quadrupolar nebula, both transitions of HNC were detected. The $V_{\mathrm{LSR}}$ of $\sim 49-53 \mathrm{~km} \mathrm{~s}^{-1}$ and $\Delta V_{1 / 2}$ of $\sim 26-27 \mathrm{~km} \mathrm{~s}^{-1}$ are consistent with those seen in the $J=3 \rightarrow 2$ spectra of $\mathrm{HCN}$ and $\mathrm{HCO}^{+}$, although the $\mathrm{HNC}$ lines are slightly narrower. From spatio-kinematic modeling, Dobrinčić et al. (2008) assign an age of $\sim 5500$ years to this source.

$N G C$ 2440: A detection of the $J=3 \rightarrow 2$ transition was made toward this source (age $\sim 3000$ years: Górny et al. 1997). The line appears to have a peak temperature of $\sim 7 \mathrm{mK}$ and a width of $\sim 50 \mathrm{~km} \mathrm{~s}^{-1}$, and is centered at $\sim 41 \mathrm{~km} \mathrm{~s}^{-1}$. This is consistent with what was seen in $\mathrm{HCN}$ and $\mathrm{HCO}^{+}$. Several sharp, narrow features may be seen near $\sim 27,41$, and $57 \mathrm{~km} \mathrm{~s}^{-1}$, which are also present in $\mathrm{HCN}$ and $\mathrm{HCO}^{+}$.

$N G C 6772 a / b$ : The $J=1 \rightarrow 0$ transition of $\mathrm{HNC}$ was detected in this large (75!"6: Tylenda et al. 2003) nebula at the central position $\left(19^{\mathrm{h}} 14^{\mathrm{m}} 37^{\mathrm{s}} .3\right.$ : $-2^{\circ} 42^{\prime} 19$ !' $\left.8 \mathrm{~J} 2000.0\right)$ provided by Milne (1973), hereafter referred to as "NGC 6772a." The observed position differs from that used by Schmidt \& Ziurys (2016) for $\mathrm{HCN}$ and $\mathrm{HCO}^{+}\left(19^{\mathrm{h}} 14^{\mathrm{m}} 36^{\mathrm{s}} .4\right.$ in R.A., $-2^{\circ} 42^{\prime} 25^{\prime \prime}$ J2000.0 from Kerber et al. (2003), hereafter known as "NGC 6772 b"). This position was also observed, but no positive detections were made. The line intensity for NGC 6772a is $T_{A}^{*} \sim 3 \mathrm{mK}$; the line width of $\sim 31 \mathrm{~km} \mathrm{~s}^{-1}$ and LSR velocity of $\sim 24 \mathrm{~km} \mathrm{~s}^{-1}$ are comparable to what is seen in HCN and CO. Ali et al. (2012) calculated a dynamical age of $\sim 11,000$ years.

K4-47: This young bipolar source contains two diametrically opposed bullets or jets of gas, which have been observed in low-ionization atomic lines. The $J=1 \rightarrow 0$ and $3 \rightarrow 2 \mathrm{HNC}$ lines were both detected in $\mathrm{K} 4-47$, with the $V_{\mathrm{LSR}}$ of $\sim-28 \mathrm{~km} \mathrm{~s}^{-1}$ closely matching that seen in $\mathrm{HCN}$ and $\mathrm{HCO}^{+}$, although the line widths may be slightly narrower. As in the case of other molecules (e.g., Edwards et al. 2014), the $J=3 \rightarrow 2$ spectrum of HNC appears to contain both a central and a plateau component, with line widths near $22 \mathrm{~km} \mathrm{~s}^{-1}$ and $37 \mathrm{~km} \mathrm{~s}^{-1}$, respectively. As described in Schmidt \& Ziurys (2016), the plateau feature may be the result of an outflow associated with bullets of gas ejected from the central star. An age of $\sim 900$ years was estimated by Corradi et al. (2000).

\subsection{Helix Positions}

$\left(130^{\prime \prime},-180^{\prime \prime}\right)$ : Two distinct velocity components are clearly visible in both the $\mathrm{HNC}$ and $\mathrm{HCN} J=1 \rightarrow 0$ spectra, one at $-10 \mathrm{~km} \mathrm{~s}^{-1}$ and the other at $-47 \mathrm{~km} \mathrm{~s}^{-1}$, as also seen in $\mathrm{CO}$, $\mathrm{HCO}^{+}$, and $\mathrm{H}_{2} \mathrm{CO}$. The $\mathrm{HNC}$ and $\mathrm{HCN}$ line widths of $2.9-3.5 \mathrm{~km} \mathrm{~s}^{-1}$ are consistent with those of these other molecules. The HCN line profiles appear noticeably wider because of the nitrogen hyperfine structure.

$\left(390^{\prime \prime},-30^{\prime \prime}\right)$ : One velocity component can be seen in both the $\mathrm{HCN}$ and the HNC spectra, with a central velocity of about $-33 \mathrm{~km} \mathrm{~s}^{-1}$. The line widths for both molecules are $\sim 6 \mathrm{~km} \mathrm{~s}^{-1}$, thus blending the hyperfine components in HCN.

$\left(-240^{\prime \prime},-100^{\prime \prime}\right)$ : A single velocity component, centered at $\sim-38 \mathrm{~km} \mathrm{~s}^{-1}$, is visible in both the HNC and HCN spectra. This feature is particularly narrow, with a $\Delta V_{1 / 2}$ of $\sim 2-3 \mathrm{~km} \mathrm{~s}^{-1}$.

$\left(-15^{\prime \prime}, 270^{\prime \prime}\right)$ : Two closely spaced velocity components can clearly be distinguished in the HNC spectrum, one centered at $-35 \mathrm{~km} \mathrm{~s}^{-1}$ and one at $-29 \mathrm{~km} \mathrm{~s}^{-1}$. The widths of each component are $\sim 3 \mathrm{~km} \mathrm{~s}^{-1}$. These velocity components are also present in the $\mathrm{HCN}$ spectrum, but are interwoven with the hyperfine structure, creating a line profile that at first glance appears to have non-LTE intensities. This profile is more accurately explained by the two velocity components, as shown in Figure 3.

$\left(-120^{\prime \prime}, 240^{\prime \prime}\right)$ : Two widely spaced velocity components are present in both the $\mathrm{HCN}$ and $\mathrm{HNC}$ spectra, at $\sim-34$ and $3 \mathrm{~km} \mathrm{~s}^{-1}$, respectively. The $\Delta V_{1 / 2}$ is $\sim 5-6 \mathrm{~km} \mathrm{~s}^{-1}$ for the $-33 \mathrm{~km} \mathrm{~s}^{-1}$ component, and around $2 \mathrm{~km} \mathrm{~s}^{-1}$ for the $3 \mathrm{~km} \mathrm{~s}^{-1}$ component. Hyperfine splitting in $\mathrm{HCN}$ is discernable in the $3 \mathrm{~km} \mathrm{~s}^{-1}$ component but the lines are completely blended in the $-34 \mathrm{~km} \mathrm{~s}^{-1}$ feature.

$\left(125^{\prime \prime}, 185^{\prime \prime}\right)$ : Three velocity components are apparent in the HNC spectrum, at $-12.5,-20$, and $-25 \mathrm{~km} \mathrm{~s}^{-1}$, respectively, with line widths $\sim 3 \mathrm{~km} \mathrm{~s}^{-1}$. Due to their relative proximity, the -20 and $-25 \mathrm{~km} \mathrm{~s}^{-1}$ features are blended. These components also appear to be present in the HCN spectrum, but the hyperfine structure complicates the line profile and the individual components are difficult to distinguish. The widths in the HCN spectrum appear to be around $3 \mathrm{~km} \mathrm{~s}^{-1}$ as well.

$\left(-435^{\prime \prime}, 75^{\prime \prime}\right)$ : Three velocity components are present in both the HCN and HNC spectra, at $\sim-14,-21$, and $-29 \mathrm{~km} \mathrm{~s}^{-1}$, respectively. These are partially blended together, creating one broad feature. The line widths range around $4-5 \mathrm{~km} \mathrm{~s}^{-1}$ for each component. The complex appearance of the HCN line profile is readily accounted for by these three components superimposed on the hyperfine structure; see Figure 3.

$\left(-300^{\prime \prime},-200^{\prime \prime}\right)$ : A component near $16-17 \mathrm{~km} \mathrm{~s}^{-1}$ is evident in both the HNC and HCN spectra, consistent with what is seen in $\mathrm{CO}, \mathrm{HCO}^{+}$, and $\mathrm{H}_{2} \mathrm{CO}$ by Zack \& Ziurys (2013). However, to adequately model the $\mathrm{HCN}$ spectrum, an additional velocity component at $\sim 21 \mathrm{~km} \mathrm{~s}^{-1}$ was included. There is a tentative detection of this component in the HNC spectrum as well. In 

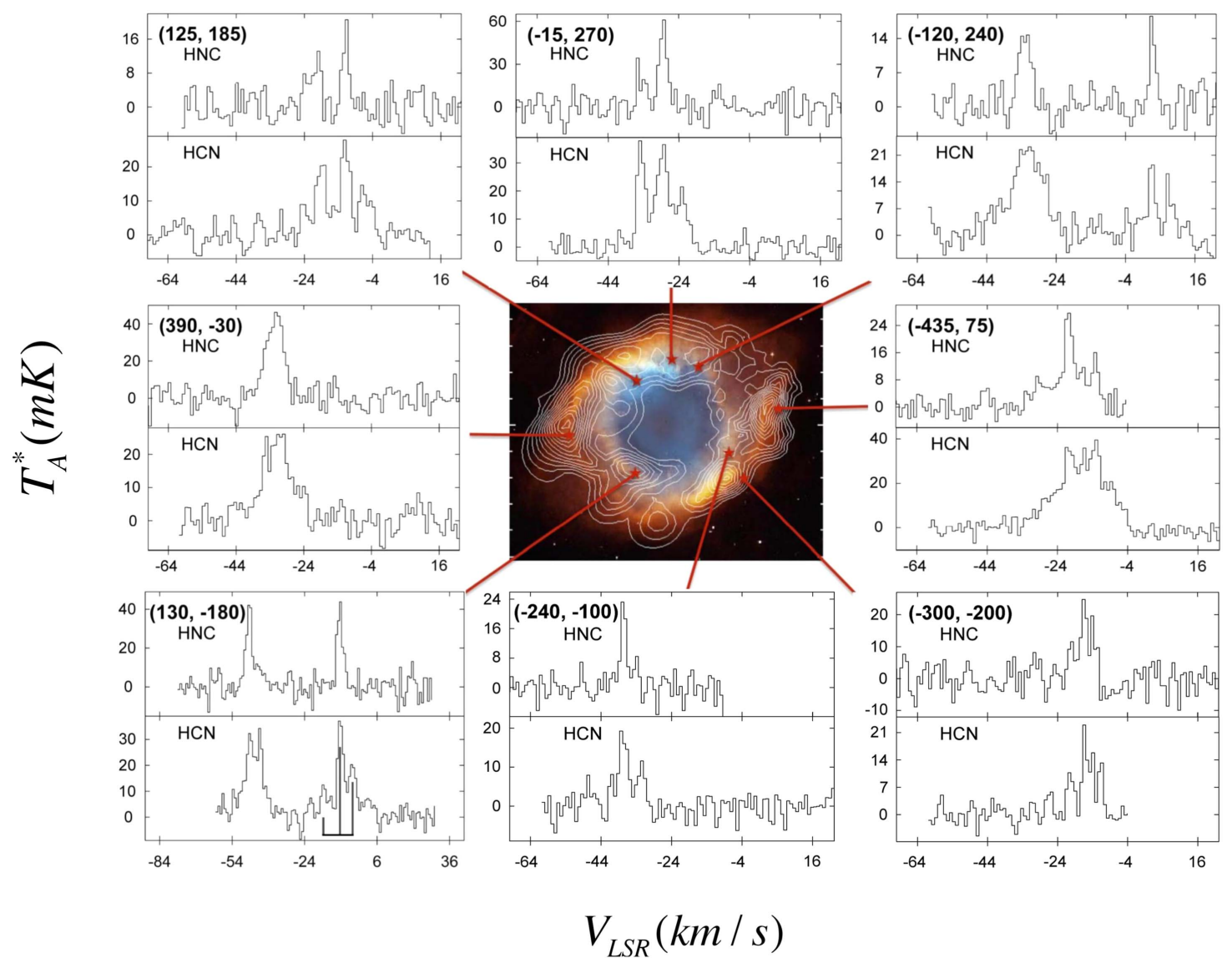

Figure 2. Spectra of the $J=1 \rightarrow 0$ transitions of $\mathrm{HNC}$ (upper panel: $90 \mathrm{GHz}$ ) and $\mathrm{HCN}$ (lower panel: $88 \mathrm{GHz}$ ) observed toward eight positions in the Helix Nebula, using the ARO ALMA prototype $12 \mathrm{~m}$. Spectral resolution is $250 \mathrm{kHz}$. The R.A. and decl. offsets for each position (in arcseconds from the central star: J2000.0: $\left.\alpha=22^{\mathrm{h}} 29^{\mathrm{m}} 38^{\mathrm{s}} .6, \delta=-20^{\circ} 50^{\prime} 18^{\prime \prime}\right)$ are displayed in the top left corner of each panel. The HCN LTE hyperfine pattern is shown beneath the $-10 \mathrm{~km} \mathrm{~s}^{-1}$ velocity component of the $\left(130^{\prime \prime},-180^{\prime \prime}\right)$ spectrum. Positions are indicated on an optical image of the Helix (NASA et al. 2003). Overlaid contours on the image indicate the peak brightness temperature of the $J=1 \rightarrow 0$ transition of $\mathrm{HCO}^{+}$(Zeigler et al. 2013). Both molecules are clearly visible in all positions, often with a complex velocity structure.

addition, there may be a third component in $\mathrm{HCN}$ near $-27 \mathrm{~km} \mathrm{~s}^{-1}$, as also observed in $\mathrm{CO}$. The line widths are typically $\sim 3 \mathrm{~km} \mathrm{~s}^{-1}$ for each component, as best can be established, though the $\sim-17 \mathrm{~km} \mathrm{~s}^{-1}$ component in HNC appears to have a broader line width of $\sim 7 \mathrm{~km} \mathrm{~s}^{-1}$.

\section{ANALYSIS}

$\mathrm{HCN}$ and $\mathrm{HNC}$ column densities were determined using the non-LTE molecular radiative transfer code, RADEX (Van der Tak et al. 2007). RADEX utilizes the Sobolev escape probability method to solve the equation of radiative transfer in order to produce line intensities for a particular molecule based on an assumed gas kinetic temperature, $\mathrm{H}_{2}$ density, and molecular column density. Considering that, at best, only two transitions were detected in a given source, the $\mathrm{H}_{2}$ density and column density were varied while the kinetic temperature was held constant at $T_{\mathrm{K}}=20 \mathrm{~K}$, a typical temperature for molecular gas in PNe (see Zack \& Ziurys 2013; Edwards \& Ziurys 2014; Edwards et al. 2014). For the remaining sources for which only a single transition had been measured, the $\mathrm{H}_{2}$ density was set to that determined from HCN by Schmidt \& Ziurys (2016). For the Helix data, both the kinetic temperature and $\mathrm{H}_{2}$ values were held fixed to those found for CO by Zack \& Ziurys (see their Table 5).
In the case of the Helix observations, there was no need to correct for beam dilution, as the source filled the beam at each position. For the other 11 survey nebulae, source filling factors were estimated in two separate ways. First, the line temperatures were corrected for beam dilution based on the nebular size estimated from the optical image, assuming a Gaussian source distribution coupled to a Gaussian beam (see Table 2). However, molecular material in $\mathrm{PNe}$ is believed to exist in dense clumps of gas and dust, not spread uniformly throughout the nebula (see, e.g., Huggins et al. 1992; Meaburn et al. 1992; Huggins \& Mauron 2002; Meixner et al. 2005). Thus, the column densities derived from these calculations apply to these knots of material, not the entirety of the PN. Therefore, as described in Schmidt \& Ziurys (2016), we also applied a "clump volume filling factor" to the data for the 11 survey $\mathrm{PNe}$, which ranged from 0.2 to 0.33 , depending on the source. The results of both calculations can be found in Table 5 for the survey sources, and those for the Helix nebula are shown in Table 6.

As shown in Table 5, the $\mathrm{H}_{2}$ densities derived from the HNC analysis for the clump $\left((0.4-3.2) \times 10^{6} \mathrm{~cm}^{-3}\right)$ and non-clump $\left((0.8-6.2) \times 10^{6} \mathrm{~cm}^{-3}\right)$ assumptions agree to within a factor of 2 , and to within a factor of 3 with the values determined from HCN by Schmidt \& Ziurys (2016). These densities are consistent with the large dipole moment of $\mathrm{HNC}(\sim 3.05$ Debye $)$ and are close to the values determined from $\mathrm{CO}, \mathrm{H}_{2} \mathrm{CO}$, and $\mathrm{CS}$ in other $\mathrm{PNe}$ 
Table 4

Observations of $\mathrm{HCN}$ and $\mathrm{HNC}(J=1 \rightarrow 0)$ Toward the Helix Nebula

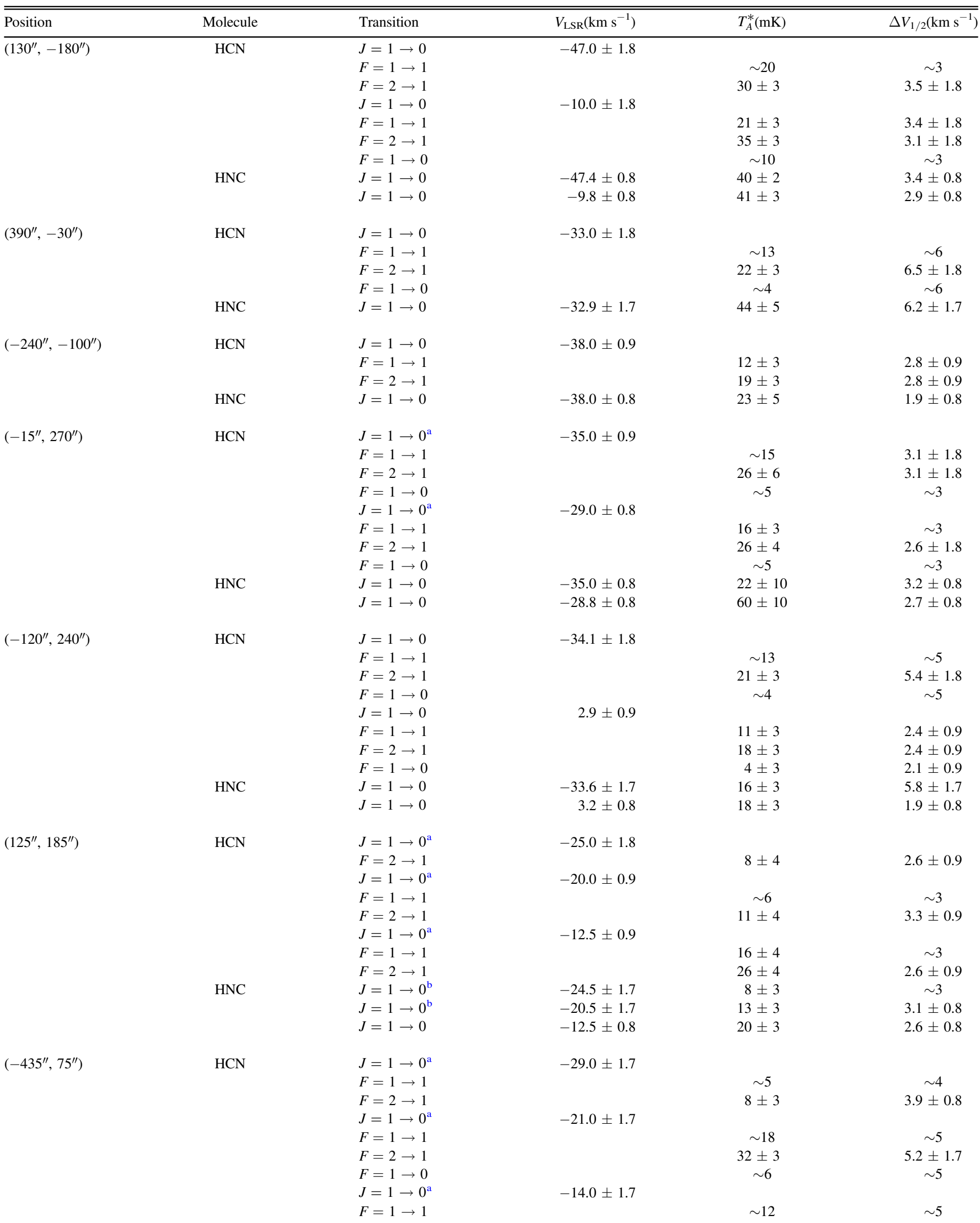


Table 4

(Continued)

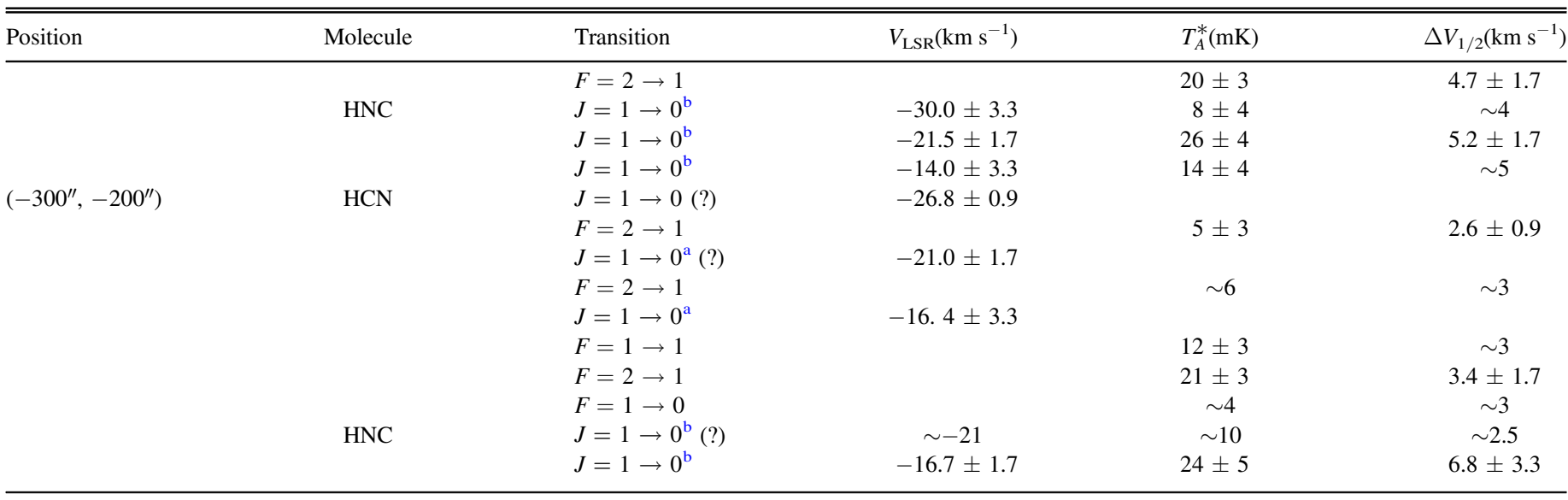

Notes.

${ }^{a}$ Hyperfine and velocity components blended.

b Blended velocity components.

(Zack \& Ziurys 2013; Edwards et al. 2014; Edwards \& Ziurys 2014). The derived column densities for HNC range between $N_{\text {tot }} \quad(\mathrm{HNC}) \sim(0.03-1.3) \times 10^{13} \mathrm{~cm}^{-2}$ and $N_{\text {tot }}$ $(\mathrm{HNC}) \sim(0.06-4.0) \times 10^{13} \mathrm{~cm}^{-2}$ for the non-clump and clump calculations - a factor of 2-7 difference. For any source for which two transitions of HNC were available, a rotational diagram was also constructed. All column densities determined from this method were within a factor of $\sim 1.4$ of their corresponding RADEX value. For the Helix, column densities fell in the range $\sim(0.2-2.4) \times 10^{12} \mathrm{~cm}^{-2}$ for $\mathrm{HCN}$ and $(0.07-1.6) \times 10^{12} \mathrm{~cm}^{-2}$ for HNC. Errors for the column density measurements were calculated through propagation of errors in the line temperatures and widths.

Using these column densities, fractional abundances with respect to $\mathrm{H}_{2}$ were calculated. $\mathrm{H}_{2}$ column densities were derived from the intensities of the $v=1 \rightarrow 0 \mathrm{~S}(1)$ transition at 2.12 microns for K4-47, K3-58, and the Helix, as such data were available (see Schmidt \& Ziurys 2016 and Zack \& Ziurys 2013). Respectively, these values are $N_{\text {tot }}\left(\mathrm{H}_{2}\right) \sim 2.9 \times 10^{20} \mathrm{~cm}^{-2}$, $\sim 1.1 \times 10^{20} \mathrm{~cm}^{-2}$, and $\sim 7.5 \times 10^{18} \mathrm{~cm}^{-2}$. For the remaining survey sources, the $\mathrm{H}_{2}$ column densities were determined from those of $\mathrm{CO}$, assuming $\mathrm{CO} / \mathrm{H}_{2} \sim 10^{-4}$ - a number consistent with other PNe (e.g., Healy \& Huggins 1990; Huggins et al. 2002; Zack \& Ziurys 2013; Edwards et al. 2014). CO column densities were taken from measurements of Schmidt \& Ziurys (2016); see their Table 4 and our Table 5.

Fractional abundances for the survey $\mathrm{PNe}$ were determined to be $f(\mathrm{HNC}) \sim(0.02-1.4) \times 10^{-7}$ (or $(0.3-6.8) \times 10^{-8}$ for the non-clump assumption-within a factor of five). For the Helix, fractional abundances for $\mathrm{HNC}$ and $\mathrm{HCN}$ were $f(\mathrm{HNC}) \sim$ $(0.09-2.2) \times 10^{-7}$ and $f(\mathrm{HCN}) \sim(0.2-3.2) \times 10^{-7}$. These values result in $[\mathrm{HCN}] /[\mathrm{HNC}] \sim 2-8$ (assuming clumping) for the survey sources (see Table 5) and $[\mathrm{HCN}] /[\mathrm{HNC}] \sim 1-4$ for the Helix (see Table 6).

\section{DISCUSSION}

\subsection{HNC Abundance and Distribution in Planetary Nebulae}

The detection of HNC in all observed targets, which represent a somewhat random sampling of the PN population, is striking, particularly considering that, just as in the case with
$\mathrm{HCN}$ and $\mathrm{HCO}^{+}$, the abundance is not negligible. The average HNC fractional abundance is $\sim 4.5 \times 10^{-8}$, including both the survey sources (assuming clumping) and the Helix positions. Furthermore, the HNC and HCN abundances across the Helix vary only by a factor of about 23 and 15 , respectively. For the $\left(-372^{\prime \prime}, 0^{\prime \prime}\right)$ position in the Helix, the data from Bachiller et al. (1997) suggest $f\left(\mathrm{HNC} / \mathrm{H}_{2}\right) \sim 3.2 \times 10^{-8}$ and $f(\mathrm{HCN} /$ $\left.\mathrm{H}_{2}\right) \sim 5.4 \times 10^{-8}$ - in line with those seen at our positions. Their measurements also suggest HNC abundances with respect to $\mathrm{H}_{2}$ of $3.5 \times 10^{-8}, 1.1 \times 10^{-8}$, and $2.2 \times 10^{-8}$ for NGC 6781, M4-9, and NGC 6720, scaling by their ${ }^{12} \mathrm{CO} /{ }^{13} \mathrm{CO}$ ratios. In NGC 6072 and IC 4406, HNC abundances of $1.9 \times 10^{-8}$ and $1.4 \times 10^{-8}$ were determined from the data of Cox et al. (1992). Previous values for the HNC abundances are comparable to those established here.

Of the Schmidt \& Ziurys (2016) survey sources, the abundance of HNC appears to be highest in K4-47 and lowest in M3-28 (assuming clumping). Similarly, K4-47 possesses a high abundance of HCN of $7.6 \times 10^{-7}$, while that in M3-28 is considerably lower at $9.8 \times 10^{-9}$. In the Helix, the $V_{\mathrm{LSR}}=-17 \mathrm{~km} \mathrm{~s}^{-1}$ component at position $\left(-300^{\prime \prime},-200^{\prime \prime}\right)$ has the highest $\mathrm{HNC}$ and $\mathrm{HCN}$ abundances, with $f$ $(\mathrm{HNC}) \sim 3.2 \times 10^{-7}$ and $f(\mathrm{HCN}) \sim 2.2 \times 10^{-7}$. Thus, the abundances of $\mathrm{HCN}$ and $\mathrm{HNC}$ generally appear to correlate.

The common precursor to both $\mathrm{HCN}$ and $\mathrm{HNC}$ in $\mathrm{PNe}$ is thought to be $\mathrm{HCNH}^{+}$(e.g., Kimura et al. 2012), as mentioned previously. This species is created by different routes, triggered by ions generated by photons or by cosmic rays. The correlation of $\mathrm{HCN}$ with $\mathrm{HNC}$ in $\mathrm{PNe}$ thus supports this hypothesis. $\mathrm{HCNH}^{+}$has yet to be detected in PNe.

Several chemical models of PNe predict abundances of HNC. For example, Redman et al. (2003) estimate the fractional abundance of $\mathrm{HNC}$ to be $\sim 1.4 \times 10^{-8}$ at 2550 years, decreasing significantly to $2.7 \times 10^{-12}$ at 6300 years and further dropping to $2.2 \times 10^{-12}$ at 10,050 years. While the abundance at 2550 years is on par with the average observed values, the predictions of Redman et al. at 6300 and 10,050 years fall far below any of the fractional abundances determined for the PNe studied here. Kimura et al. (2012) predict $\mathrm{HNC}$ abundances as high as $10^{-9}$, in somewhat better agreement, though they did not study evolution with age. 

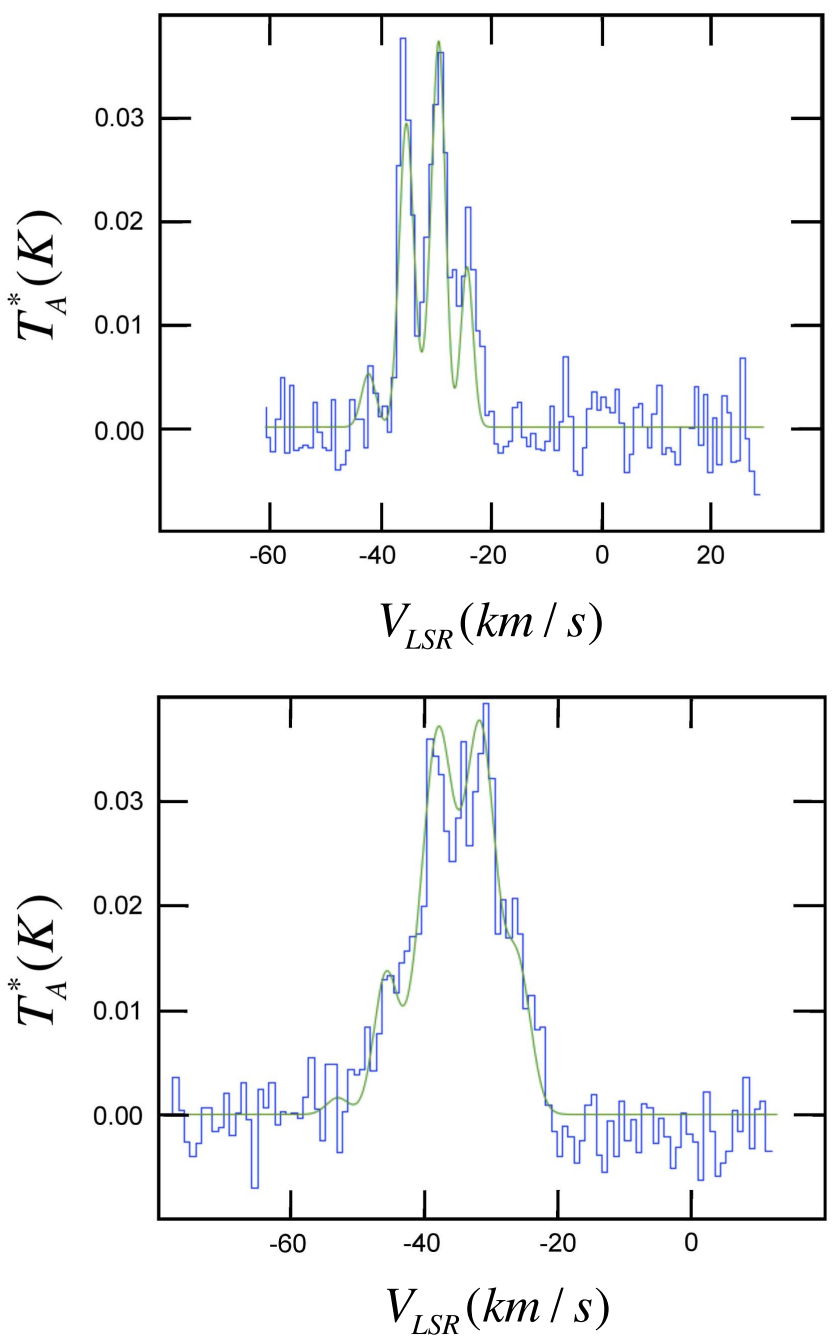

Figure 3. Representative Gaussian modeling (green or grayscale) of the velocity and hyperfine structure of the $\operatorname{HCN}(J=1 \rightarrow 0)$ line profiles at positions $\left(-15^{\prime \prime}, 270^{\prime \prime}\right)$ and $\left(-435^{\prime \prime}, 75^{\prime \prime}\right)$ in the Helix Nebula (upper and lower panels). The modeling assumes LTE ratios for the three hyperfine lines (see Figure 2). The $\left(-15^{\prime \prime}, 270^{\prime \prime}\right)$ spectrum was fit with two velocity components at -29 and $-35 \mathrm{~km} \mathrm{~s}^{-1}$; the $\left(-435^{\prime \prime}, 75^{\prime \prime}\right)$ data were analyzed with three such components at $-29,-21$, and $-14 \mathrm{~km} \mathrm{~s}^{-1}$. Velocity components were deduced from $\mathrm{HNC}$ and previous $\mathrm{CO}$ and $\mathrm{HCO}^{+}$data from Zack \& Ziurys (2013).

Our data also indicate that there is no strong variation of HNC abundance with nebular age, as illustrated in Figure 4. This figure plots the HNC abundance (cyan circles), assuming clumping, as a function of source age, along with those for $\mathrm{HCN}$ and $\mathrm{HCO}^{+}$from Schmidt \& Ziurys (in red and blue circles, respectively). Also plotted are abundances in other sources, indicated by stars. Model predictions from Redman et al. are represented as triangles. As shown in the figure, the observed values fall in the range $\sim 10^{-8}-10^{-7}$, and do not change significantly from about 1000 to 11,000 years. Although there are uncertainties in the ages of the nebulae, the trend is very clear. The models predict a sharp decrease of $10^{4}$ by 6000 years for HNC, with a steady abundance of $10^{-12}$ thereafter. Indeed, these findings support the idea that clumped material shields the molecules from incoming radiation more effectively than predicted by models, and/or that there is more active chemical production ongoing in PNe than expected, as noted by Schmidt \& Ziurys (2016).
$\mathrm{HNC}$ is a prominent molecule in C-rich AGB envelopes, including IRC+10216, IRAS 15194-5115, IRAS 15082-4808, IRAS 07454-7112, CIT 6, AFGL 3068, and IRC+40540 (Woods et al. 2003). The abundances of HNC in these objects ranged from $(0.2-3.3) \times 10^{-7}$. In contrast, Smith et al. (2015) found $f\left(\mathrm{HNC} / \mathrm{H}_{2}\right) \leqslant(0.5-2.9) \times 10^{-8}$ for the envelopes of C-stars II Lup, RAFGL 4211, and AI Vol. HNC has also been identified in a number of proto-planetary nebulae (PPNe). Morris et al. (1987) derived an HNC abundance of $1.8 \times 10^{-7}$ for the protoplanetary Calabash Nebula, while in CRL 2688, Zhang et al. (2013) found $f\left(\mathrm{HNC} / \mathrm{H}_{2}\right) \sim 5.7 \times 10^{-7}$ - higher than those of the PNe observed here. For CRL 618, the abundance is estimated to be in the range $1.9 \times 10^{-7}$ $1.9 \times 10^{-6}$ (Sopka et al. 1989; Bujarrabal et al. 1994). Comparing these HNC abundances with those measured in $\mathrm{PNe}$, the values on average decrease slightly from the AGB stage through the PN phase.

\section{2. $[\mathrm{HNC}] /[\mathrm{HCN}]$ Ratio with Nebular Age}

The $[\mathrm{HCN}] /[\mathrm{HNC}]$ ratios established here fall within the range 1-8 for all sources. The ratio appears to be highest in $\mathrm{K} 3-$ 17, a young PN, and lowest in M3-55, which is middle-aged. These values are consistent with those found in other PNe. Using the data of Bachiller et al. (1997), NGC 6720, M4-9, and NGC 6781 have ratios ranging from $\sim 1.4-4.5$, while several compact $\mathrm{PNe}$ are estimated to have $[\mathrm{HCN}] /[\mathrm{HNC}] \sim 2.5$ (Josselin \& Bachiller 2003). The HNC data in the latter case have limited sensitivity. Regardless, it is clear that the ratio is remarkably constant, given the different types of nebulae and their physical characteristics.

There may be a slight correlation of the $[\mathrm{HCN}] /[\mathrm{HNC}]$ ratio with stellar temperature, which in principle could convert some $\mathrm{HNC}$ to $\mathrm{HCN}$. Hb 5 has a ratio of 7 and a high temperature for its central star of $T_{*} \geqslant 150,000 \mathrm{~K}$ (Pottasch \& Surendiranath 2007). On the other hand, NGC 2440 and M3-28 both have ratios near 4, with respective stellar temperatures of $T_{*} \sim 188,400 \mathrm{~K}$ (Heap 1987) and $T_{*} \sim 130,500 \mathrm{~K}$ (PreiteMartinez et al. 1989). Temperatures for the other sources are not known. Therefore, it is difficult to determine such a correlation. More data are clearly needed.

Figure 5 shows a plot of $[\mathrm{HCN}] /[\mathrm{HNC}]$ versus nebular age. There does not appear to be any significant variation in the ratio with age. There are no sharp deviations in the general trend.

The $[\mathrm{HCN}] /[\mathrm{HNC}]$ ratios found in this work are lower than those typically seen in AGB stars. Woods et al. (2003) measured $[\mathrm{HCN}] /[\mathrm{HNC}]$ ratios of $\sim 40-300$ for the seven carbon stars observed. The value for IRC +10215 is near 300 . The ratio apparently drops in the PPN stage. The ratio for CRL2688 is $\sim 1$ (Zhang et al. 2013), while the value in PPN IRAS $16594-4656$ is about 3.4 (Woods et al. 2005). Herpin \& Cernicharo (2000) found relatively equal column densities for $\mathrm{HCN}$ and $\mathrm{HNC}$ in the PPN CRL618, implying [HCN]/ $[\mathrm{HNC}] \sim 1$, while Morris et al. (1987) found a ratio of $\sim 2.2$ for the Calabash Nebula.

The rise in $\mathrm{HNC}$ with respect to $\mathrm{HCN}$ in the PPN phase has been attributed to an increase in ionization. As discussed, HCN is effectively produced by LTE chemistry in the inner envelope of AGB stars, while HNC is not, thereby producing the high $[\mathrm{HCN}] /[\mathrm{HNC}]$ ratios often observed. In the PPN stage, the increase in ionization drives reactions that lead to the formation of $\mathrm{HNC}$ through the effective intermediate, $\mathrm{HCNH}^{+}$. The ion $\mathrm{HCNH}^{+}$can form from several different ion-neutral reactions 
Table 5

Column Densities and Fractional Abundances for Survey PNe

\begin{tabular}{|c|c|c|c|c|c|c|}
\hline Source & & $n\left(\mathrm{H}_{2}\right)\left(\mathrm{cm}^{-3}\right)$ & $N_{\text {tot }}\left(\mathrm{cm}^{-2}\right)$ & $N_{\text {tot }}(\mathrm{CO})\left(\mathrm{cm}^{-2}\right)$ & $\mathrm{X}_{\mathrm{i}} / \mathrm{H}_{2}$ & {$[\mathrm{HCN}] /[\mathrm{HNC}]$} \\
\hline \multirow[t]{2}{*}{$\mathrm{Hb} 5$} & Clump & $4.3 \mathrm{e} 5$ & $5.3 \pm 2.0 \times 10^{12}$ & $4.3 \pm 1.5 \times 10^{15}$ & $1.2 \pm 0.3 \times 10^{-7}$ & 7.4 \\
\hline & Non-clump & $7.6 \mathrm{e} 5$ & $1.7 \pm 0.7 \times 10^{12}$ & $2.5 \pm 2.4 \times 10^{15}$ & $6.8 \pm 3.6 \times 10^{-8}$ & 7.1 \\
\hline \multirow[t]{2}{*}{ K3-17 } & Clump & $1.2 \mathrm{e} 6$ & $3.4 \pm 1.9 \times 10^{13}$ & $3.9 \pm 2.0 \times 10^{17}$ & $8.7 \pm 3.3 \times 10^{-9}$ & 7.9 \\
\hline & Non-clump & $1.7 \mathrm{e} 6$ & $5.0 \pm 2.8 \times 10^{12}$ & $6.5 \pm 5.0 \times 10^{16}$ & $7.7 \pm 3.7 \times 10^{-9}$ & 4.8 \\
\hline \multirow[t]{2}{*}{ K3-45 } & Clump & $1.1 \mathrm{e} 6$ & $1.4 \pm 0.5 \times 10^{13}$ & $2.9 \pm 1.6 \times 10^{17}$ & $4.8 \pm 1.6 \times 10^{-9}$ & 3.8 \\
\hline & Non-clump & $9.4 \mathrm{e} 5$ & $5.3 \pm 1.8 \times 10^{12}$ & $6.7 \pm 1.9 \times 10^{16}$ & $7.9 \pm 1.7 \times 10^{-9}$ & 3.2 \\
\hline \multirow[t]{2}{*}{$\mathrm{K} 3-58$} & Clump & $7.5 \mathrm{e} 5$ & $1.2 \pm 0.3 \times 10^{13}$ & $\ldots$ & $1.1 \pm 0.1 \times 10^{-7}$ & 2.1 \\
\hline & Non-clump & $1.0 \mathrm{e} 6$ & $2.4 \pm 0.7 \times 10^{12}$ & $\ldots$ & $2.2 \pm 0.3 \times 10^{-8}$ & 2.1 \\
\hline \multirow[t]{2}{*}{ M1-7 } & Clump & 1.1e6 & $2.0 \pm 1.0 \times 10^{13}$ & $8.3 \pm 7.4 \times 10^{17}$ & $2.4 \pm 1.2 \times 10^{-9}$ & 5.8 \\
\hline & Non-clump & $1.1 \mathrm{e} 6$ & $6.6 \pm 3.2 \times 10^{12}$ & $1.1 \pm 0.2 \times 10^{17}$ & $6.0 \pm 1.6 \times 10^{-9}$ & 5.5 \\
\hline \multirow[t]{2}{*}{ M3-28 } & Clump & $9.5 \mathrm{e} 5$ & $2.2 \pm 0.5 \times 10^{13}$ & $9.3 \pm 7.5 \times 10^{17}$ & $2.4 \pm 1.0 \times 10^{-9}$ & 4.1 \\
\hline & Non-clump & $1.3 \mathrm{e} 6$ & $4.4 \pm 1.0 \times 10^{12}$ & $1.3 \pm 0.3 \times 10^{17}$ & $3.4 \pm 0.6 \times 10^{-9}$ & 4.1 \\
\hline \multirow[t]{2}{*}{ M3-55 } & Clump & $1.1 \mathrm{e} 6$ & $2.3 \pm 0.7 \times 10^{13}$ & $1.1 \pm 0.5 \times 10^{17}$ & $2.1 \pm 0.6 \times 10^{-8}$ & 2.0 \\
\hline & Non-clump & $1.1 \mathrm{e} 6$ & $4.5 \pm 1.3 \times 10^{12}$ & $2.7 \pm 2.1 \times 10^{16}$ & $1.7 \pm 0.7 \times 10^{-8}$ & 1.0 \\
\hline \multirow[t]{2}{*}{ M4-14 } & Clump & $1.6 \mathrm{e} 6$ & $7.7 \pm 3.0 \times 10^{12}$ & $3.6 \pm 1.2 \times 10^{16}$ & $2.1 \pm 0.5 \times 10^{-8}$ & 5.1 \\
\hline & Non-clump & $2.1 \mathrm{e} 6$ & $1.6 \pm 0.7 \times 10^{12}$ & $1.3 \pm 0.8 \times 10^{16}$ & $1.2 \pm 0.4 \times 10^{-8}$ & 3.0 \\
\hline \multirow[t]{2}{*}{ NGC 2440} & Clump & $3.2 \mathrm{e} 6$ & $2.6 \pm 1.3 \times 10^{12}$ & $7.2 \pm 4.8 \times 10^{15}$ & $3.6 \pm 1.5 \times 10^{-8}$ & 4.7 \\
\hline & Non-clump & $6.2 \mathrm{e} 6$ & $7.9 \pm 3.7 \times 10^{11}$ & $7.2 \pm 6.7 \times 10^{15}$ & $1.1 \pm 0.6 \times 10^{-8}$ & 4.6 \\
\hline \multirow[t]{2}{*}{ NGC 6772a } & Clump & $1.2 \mathrm{e} 6$ & $5.6 \pm 3.7 \times 10^{11}$ & $2.5 \pm 1.1 \times 10^{15}$ & $2.2 \pm 0.9 \times 10^{-8}$ & 4.1 \\
\hline & Non-clump & $2.9 \mathrm{e} 6$ & $2.9 \pm 1.9 \times 10^{11}$ & $1.5 \pm 0.3 \times 10^{15}$ & $1.9 \pm 0.7 \times 10^{-8}$ & 3.5 \\
\hline \multirow[t]{2}{*}{ K4-47 } & Clump & $2.1 \mathrm{e} 6$ & $4.0 \pm 1.7 \times 10^{13}$ & $\ldots$ & $1.4 \pm 0.2 \times 10^{-7}$ & 5.5 \\
\hline & Non-clump & $2.1 \mathrm{e} 6$ & $1.3 \pm 0.6 \times 10^{13}$ & $\ldots$ & $4.5 \pm 0.7 \times 10^{-8}$ & 4.9 \\
\hline
\end{tabular}

Table 6

Column Densities and Fractional Abundances for Helix Positions

\begin{tabular}{|c|c|c|c|c|c|c|}
\hline Position & $V_{\mathrm{LSR}}\left(\mathrm{km} \mathrm{s}^{-1}\right)$ & $N_{\text {tot }}(\mathrm{HCN})\left(10^{11} \mathrm{~cm}^{-2}\right)$ & $N_{\text {tot }}(\mathrm{HNC})\left(10^{11} \mathrm{~cm}^{-2}\right)$ & $\mathrm{X}_{\mathrm{i}} / \mathrm{H}_{2}(\mathrm{HCN})\left(10^{-8}\right)$ & $\mathrm{X}_{\mathrm{i}} / \mathrm{H}_{2}(\mathrm{HNC})\left(10^{-8}\right)$ & {$[\mathrm{HCN}] /[\mathrm{HNC}]$} \\
\hline \multirow[t]{2}{*}{$\left(130^{\prime \prime},-180^{\prime \prime}\right)$} & -47 & $3.7 \pm 1.2$ & $2.3 \pm 0.2$ & $4.9 \pm 0.9$ & $3.1 \pm 0.3$ & 1.6 \\
\hline & -10 & $3.8 \pm 2.1$ & $2.0 \pm 0.6$ & $5.1 \pm 1.4$ & $2.7 \pm 0.4$ & 1.9 \\
\hline$\left(390^{\prime \prime},-30^{\prime \prime}\right)$ & -33 & $9.0 \pm 0.9$ & $6.6 \pm 1.3$ & $12.0 \pm 1.0$ & $8.8 \pm 1.0$ & 1.4 \\
\hline$\left(-240^{\prime \prime},-100^{\prime \prime}\right)$ & -38 & $1.8 \pm 0.6$ & $0.7 \pm 0.4$ & $2.4 \pm 0.4$ & $0.9 \pm 0.3$ & 2.7 \\
\hline \multirow[t]{2}{*}{$\left(-15^{\prime \prime}, 270^{\prime \prime}\right)$} & -35 & $4.4 \pm 2.2$ & $1.6 \pm 0.8$ & $5.9 \pm 1.5$ & $2.1 \pm 0.5$ & 2.8 \\
\hline & -29 & $1.9 \pm 1.4$ & $2.5 \pm 0.9$ & $2.5 \pm 0.9$ & $3.3 \pm 0.6$ & 0.8 \\
\hline \multirow[t]{2}{*}{$\left(-120^{\prime \prime}, 240^{\prime \prime}\right)$} & -33 & $6.2 \pm 2.4$ & $2.1 \pm 0.9$ & $8.3 \pm 1.7$ & $2.8 \pm 0.6$ & 3.0 \\
\hline & 4 & $2.6 \pm 1.1$ & $0.9 \pm 0.4$ & $3.5 \pm 0.8$ & $1.2 \pm 0.3$ & 2.9 \\
\hline \multirow[t]{3}{*}{$\left(125^{\prime \prime}, 185^{\prime \prime}\right)$} & -25 & $13.0 \pm 6.8$ & $3.9 \pm 1.1$ & $17.3 \pm 4.6$ & $5.2 \pm 0.8$ & 3.3 \\
\hline & -20 & $22.9 \pm 9.4$ & $6.6 \pm 2.2$ & $30.5 \pm 6.7$ & $8.8 \pm 1.6$ & 3.5 \\
\hline & -13 & $11.7 \pm 1.4$ & $2.9 \pm 0.4$ & $15.6 \pm 1.4$ & $3.9 \pm 0.4$ & 4.0 \\
\hline \multirow[t]{3}{*}{$\left(-435^{\prime \prime}, 75^{\prime \prime}\right)$} & -29 & $1.6 \pm 0.4$ & $0.7 \pm 0.2$ & $2.1 \pm 0.3$ & $0.9 \pm 0.1$ & 2.3 \\
\hline & -21 & $18.3 \pm 4.0$ & $5.0 \pm 1.7$ & $24.4 \pm 3.1$ & $6.7 \pm 1.2$ & 3.7 \\
\hline & -13 & $16.1 \pm 1.6$ & $3.8 \pm 0.2$ & $21.4 \pm 1.7$ & $5.1 \pm 0.4$ & 4.2 \\
\hline \multirow[t]{3}{*}{$\left(-300^{\prime \prime},-200^{\prime \prime}\right)$} & -27 & $4.2 \pm 1.3$ & $\cdots$ & $5.6 \pm 0.9$ & $\cdots$ & $\cdots$ \\
\hline & -21 & $5.8 \pm 2.4$ & $2.5 \pm 0.3$ & $7.7 \pm 1.7$ & $3.3 \pm 0.3$ & 2.3 \\
\hline & -17 & $23.9 \pm 3.6$ & $16.4 \pm 4.7$ & $31.9 \pm 3.2$ & $21.8 \pm 3.5$ & 1.5 \\
\hline
\end{tabular}

(e.g., Daniel et al. 2012):

$$
\begin{gathered}
\mathrm{HCO}^{+}+\mathrm{HCN} \rightarrow \mathrm{HCNH}^{+}+\mathrm{CO} \\
\mathrm{C}_{2} \mathrm{H}_{3}^{+}+\mathrm{HCN} \rightarrow \mathrm{HCNH}^{+}+\mathrm{C}_{2} \mathrm{H}_{2} \\
\mathrm{H}_{3}^{+}+\mathrm{HCN} \rightarrow \mathrm{HCNH}^{+}+\mathrm{H}_{2} .
\end{gathered}
$$

HNC is then produced via dissociative electron recombination, Equation (1), where the branching ratio is estimated to be unity (Talbi 1999). Additionally, increased UV radiation in the PPN and PN phases is effective in creating radical species (Daniel et al. 2012). HNC may also form through the reaction

$$
\mathrm{C}+\mathrm{NH}_{2} \rightarrow \mathrm{HNC}+\mathrm{H} \text {. }
$$

Over time, these reactions skew the $[\mathrm{HCN}] /[\mathrm{HNC}]$ ratio toward unity. Observations indicate that the rise of HNC over
$\mathrm{HCN}$ occurs principally in PPNe. The ratio then appears to "freeze out" into the PN phase. The striking consistency of the $[\mathrm{HCN}] /[\mathrm{HNC}]$ ratio across the Helix Nebula is evidence of such a freeze-out.

\subsection{Comparison with Diffuse Cloud Abundances}

Liszt \& Lucas (2001) and Liszt et al. (2006) observed both $\mathrm{HCN}$ and HNC (among other molecules) in a set of seven diffuse clouds observed against background extragalactic sources. The $\mathrm{HCN}$ abundances ranged from $(0.14-0.5) \times 10^{-8}$ with respect to $\mathrm{H}_{2}$, while the HNC abundances fell between $(0.03-0.10) \times 10^{-8}$. The authors noted both $\mathrm{HCN}$ and $\mathrm{HNC}$ were much more plentiful in diffuse clouds than predicted by models of quiescent lowdensity gas-phase chemistry. The reaction rates necessary to form 


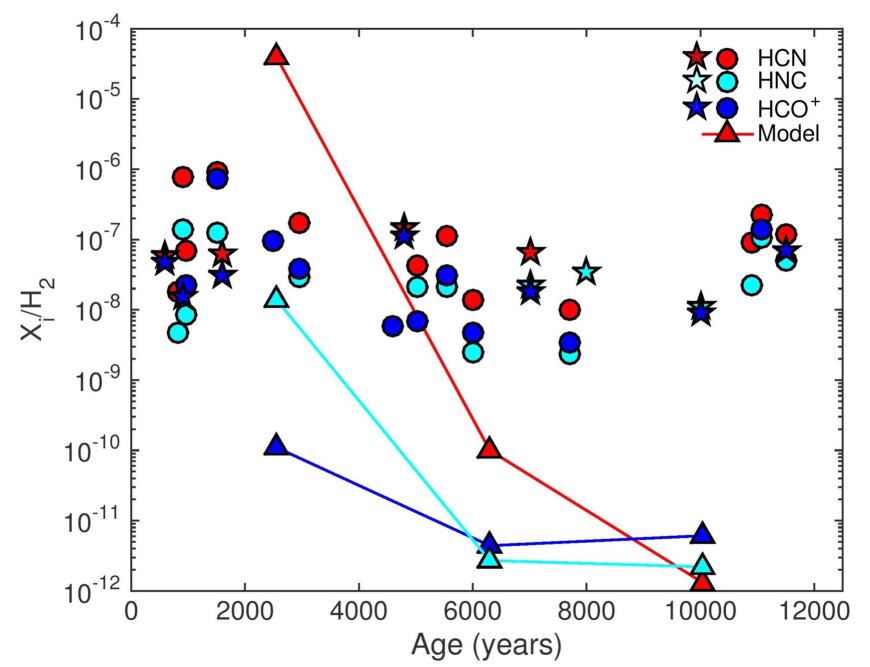

Figure 4. Plot of fractional abundances of $\mathrm{HCN}$ (red), $\mathrm{HCO}^{+}$(blue), and $\mathrm{HNC}$ (cyan) relative to $\mathrm{H}_{2}$ in PNe vs. nebular age in years. All abundances assume clumping. Circles represent data from this work (HNC) and Schmidt \& Ziurys (2016): $\mathrm{HCN}, \mathrm{HCO}^{+}$. Red and blue stars indicate measurements for $\mathrm{HCN}$ and $\mathrm{HCO}^{+}$for NGC 7027, NGC 6537, M2-48, NGC 6853, and NGC 6720 from previous works (see text and Schmidt \& Ziurys 2016). The cyan stars designate HNC abundances for NGC 6720, M4-9, and NGC 6781 from Bachiller et al (1997) (see text for details). The red, blue, and cyan triangles (dashed lines) designate the fractional abundances for $\mathrm{HCN} \mathrm{HCO}^{+}$, and $\mathrm{HNC}$, respectively, from the model of Redman et al. (2003). The model predicts a sharp decrease in the fractional abundances of $\mathrm{HNC}$ and $\mathrm{HCN}$ as a function of time, and significantly lower values than observed. The measured abundances of all three molecules appear to stay relatively constant across the $\sim 10,000$ year lifetime of PNe.

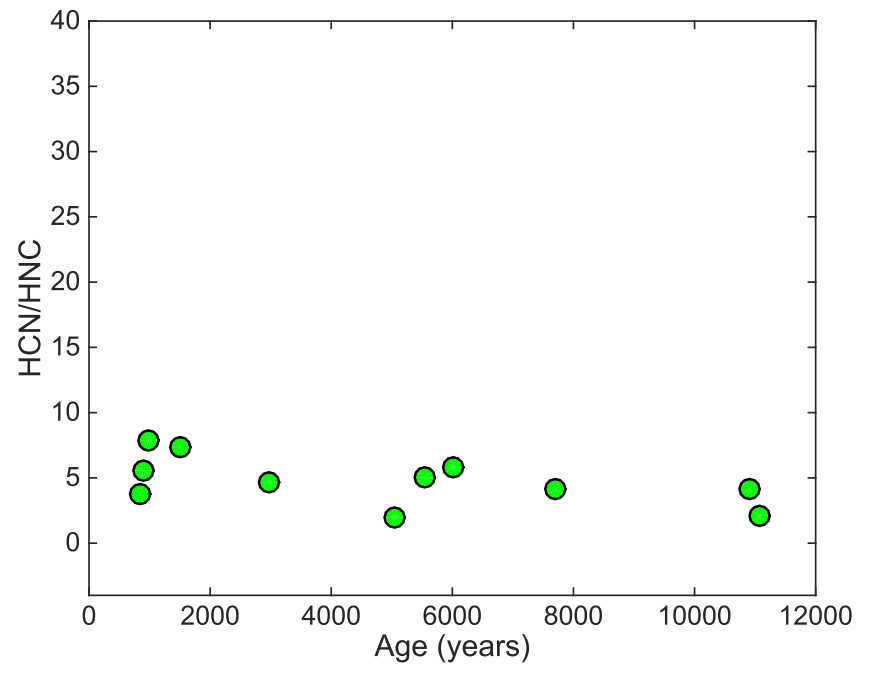

Figure 5. Plot of $[\mathrm{HCN}] /[\mathrm{HNC}]$ ratio (assuming clumping) vs. PN age in years. The ratio appears to be relatively stable over $\sim 10,000$ years, indicating that, by the PN stage, the abundances of HCN and HNC have "frozen out" from those generated in the PPNe phase.

$\mathrm{CN}$-bearing molecules are simply too slow to explain the observed abundances. Based on their measurements, Liszt et al. (2006) estimated an average $[\mathrm{HCN}] /[\mathrm{HNC}]$ ratio of 5.

The abundances for HNC in diffuse clouds are 1-2 orders of magnitude lower than the abundances measured for $\mathrm{PNe}$. Furthermore, the average $[\mathrm{HCN}] /[\mathrm{HNC}]$ ratio found for diffuse clouds is remarkably close to that found in PNe: 5 versus $1-8$. These results bolster the argument made in Schmidt \& Ziurys (2016) and Ziurys et al. $(2015,2016)$ that the polyatomic molecules observed in diffuse clouds originate in PNe ejecta.
The clumps of molecular material, mixed with dust, disperse into the diffuse ISM and later coalesce into clouds.

\section{CONCLUSIONS}

This work, in conjunction with past observations, has demonstrated that $\mathrm{HNC}$ is prevalent in many $\mathrm{PNe}$, as has been also found for $\mathrm{HCN}$ and $\mathrm{HCO}^{+}$. Both $\mathrm{HNC}$ abundances and the $[\mathrm{HCN}] /[\mathrm{HNC}]$ ratio measured in PNe are comparable to those found in PPNe, suggesting that much of the chemistry altering AGB abundances occurs in the protoplanetary phase. The lack of variation in the $[\mathrm{HCN}] /[\mathrm{HNC}]$ ratio across eight positions in the Helix is further evidence for the predominance of PPNe synthesis. This study also strongly bolsters the concept that molecular abundances appear to remain relatively constant across the $\sim 10,000$ year lifespan of PNe, as argued in Schmidt \& Ziurys (2016). Finally, fractional abundances for HNC were found to be 1-2 magnitudes higher than those seen in diffuse clouds, supporting the idea that PNe provide molecular material to the ISM.

This research was supported by NSF grants AST-1515568, AST-1140030, and by the NASA NExSS program via grant NNX13ZDA017C. The Kitt Peak $12 \mathrm{~m}$ and the SMT are operated by the Arizona Radio Observatory (ARO), University of Arizona, with partial support through the NSF University Radio Observatories (URO) program (AST-1140030).

\section{REFERENCES}

Acker, A., Marcout, J., Ochsenbein, F., Stenholm, B., \& Tylenda, R. 1992, The Strasbourg-ESO Catalogue of Galactic Planetary Nebulae. Parts 1 and 2 (Garching: European Southern Observatory)

Ali, A., Sabin, L, Snaid, S., \& Basurah, H. M. 2012, A\&A, 541, A98

Bachiller, R., Forveille, T., Huggins, P. J., \& Cox, P. 1997, A\&A, 324, 1123

Bujarrabal, V., Fuente, A., \& Omont, A. 1994, A\&A, 285, 247

Cami, J., Bernard-Salas, J., Peeters, E., \& Malek, S. E. 2010, Sci, 329, 1180

Corradi, R. L. M., Gonçalves, R., Villaver, E., et al. 2000, ApJ, 535, 823

Cox, P. 1997, in IAU Symp. 178, Molecules in Astrophysics: Probes \& Processes, ed. E. F. van Dishoeck (Dordrecht: Kluwer), 477

Cox, P., Omont, A., Huggins, P. J., Bachiller, R., \& Forveille, T. 1992, A\&A, 266, 420

Daniel, F., Agúndez, M., Cernicharo, J., et al. 2012, A\&A, 542, A37

Dobrinčić, M., Villaver, E., Guerrero, M. A., \& Manchado, A. 2008, AJ, 135, 2199

Dufour, R. J., Kwitter, K. B., Shaw, R. A., et al. 2015, ApJ, 803, 23

Edwards, J. L., Cox, E. G., \& Ziurys, L. M. 2014, ApJ, 791, 79

Edwards, J. L., \& Ziurys, L. M. 2013, ApJL, 770, L5

Edwards, J. L., \& Ziurys, L. M. 2014, ApJL, 794, L27

García-Hernández, D. A., Iglesias-Groth, S., Acosta-Pulido, J. A., et al. 2011, ApJL, 737, L30

García-Hernández, D. A., Manchado, A., García-Lario, P., et al. 2010, ApJL, 724, L39

García-Hernández, D. A., Villaver, E., García-Lario, P., et al. 2012, ApJ, 760,107

Giammanco, C., Sale, S. E., Corradi, R. L. M., et al. 2011, A\&A, 525, A58

Górny, S. K., Stasiñska, G., \& Tylenda, R. 1997, A\&A, 318, 256

Healy, A. P., \& Huggins, P. J. 1990, AJ, 100, 511

Heap, S. R. 1987, Natur, 326, 571

Herpin, F., \& Cernicharo, J. 2000, ApJL, 530, L129

Hora, J. L, Latter, W. B., \& Deutsch, L. K. 1999, ApJS, 124, 195

Howe, D. A., Hartquist, T. W., \& Williams, D. A. 1994, MNRAS, 271, 811

Huggins, P. J., Bachiller, R., Cox, P., \& Forveille, T. 1992, ApJL, 401, L43

Huggins, P. J., Bachiller, R., Cox, P., \& Forveille, T. 1996, A\&A, 315, 284

Huggins, P. J., Bachiller, R., Planesas, P., Forveille, T., \& Cox, P. 2005, ApJS, 160,272

Huggins, P. J., Forveille, T., Bachiller, R., et al. 2002, ApJL, 573, L55

Huggins, P. J., \& Healy, A. P. 1989, ApJ, 346, 201

Huggins, P. J., \& Mauron, N. 2002, A\&A, 393, 273

Irvine, W. M., \& Schloerb, F. P. 1984, ApJ, 282, 516

Josselin, E., \& Bachiller, R. 2003, A\&A, 397, 659 
Kerber, F., Mignani, R. P, Guglielmetti, F., \& Wiecenec, A. 2003, A\&A, 408, 1029

Kimura, R. K., Gruenwald, R., \& Aleman, I. 2012, A\&A, 541, A112

Kwok, S. 2000, The Origin and Evolution of Planetary Nebulae (Cambridge: Cambridge Univ. Press)

Likkel, L., Dinerstein, H. L., Lester, D. F., Kindt, A., \& Bartig, K. 2006, AJ, 131,1515

Liszt, H., \& Lucas, R. 2001, A\&A, 370, 576

Liszt, H. S., Lucas, R., \& Pety, J. 2006, A\&A, 448, 253

López, J. A., Garciá-Diáz, M. T., Steffen, W., Riesgo, H., \& Richer, M. G. 2012, ApJ, 750, 131

Meaburn, J., Walsh, J. R., Clegg, R. E. S., et al. 1992, MNRAS, 255, 177

Meixner, M., McCullough, P., Hartman, J., Son, M., \& Speck, A. 2005, AJ, 130, 1784

Milne, D. K. 1973, AJ, 78, 239

Morris, M., Guilloteau, S., Lucas, R., \& Omont, A. 1987, ApJ, 321, 888

NASA, NOAO, ESA, the Hubble Helix Nebula Team, M. Meixner (STScI), \& Rector, T.A. (NRAO). 2003, Iridescent Glory of the Helix Nebula. Retrieved from: http://hubblesite.org/newscenter/archive/releases/2003/11/image/a/

Otsuka, M., Kemper, F., Hyung, S., et al. 2013, ApJ, 764, 77

Patriarchi, P., \& Perinotto, M. 1994, A\&A, 287, 585

Penzias, A. A., Jefferts, K. B., \& Wilson, R. W. 1971, ApJ, 165, 229

Phillips, J. P., \& Márquez-Lugo, R. A. 2011, RMxAA, 47, 83

Pottasch, S. R., \& Bernard-Salas, J. 2010, A\&A, 517, A95

Pottasch, S. R., \& Surendiranath, R. 2007, A\&A, 462, 179

Preite-Martinez, A., Acker, A., Köppen, J., \& Stenholm, B. 1989, A\&AS, 81, 309
Redman, M. P., Viti, S., Cau, P., \& Williams, D. A. 2003, MNRAS, 345, 1291 Schilke, P., Walmsley, C. M., Pineau des Forets, G., et al. 1992, A\&A, 256,595

Schmidt, D. R., \& Ziurys, L. M. 2016, ApJ, 817, 175

Smith, C. L., Zijlstra, A. A., \& Fuller, G. A. 2015, MNRAS, 454, 177

Sopka, R. J., Olofsson, H., Johansson, L. E. B., Nguyen, Q. R., \& Zuckerman, B. 1989, A\&A, 210, 78

Speck, A., Meixner, M., Jacoby, G. H., \& Knezek, P. M. 2003, PASP, 115, 170

Talbi, D. 1999, CPL, 312, 298

Tenenbaum, E. D., Milam, S. N., Woolf, N. J., \& Ziurys, L. M. 2009, ApJL, 704, L108

Tylenda, R., Siódmiak, N., Górny, S. K., Corradi, R. L. M., \& Schwarz, H. E. 2003, A\&A, 405, 627

Van der Tak, F. F. S., Black, J. H., Schöier, F. L., Jansen, D. J., \& van Dishoeck, E. F. 2007, A\&A, 468, 627

Wilson, W. J., Schwartz, P. R., Epstein, E. E., et al. 1974, ApJ, 191, 357

Woods, P. M., Nyman, L. A., Schöier, F. L., et al. 2005, A\&A, 429, 977

Woods, P. M., Schöier, F. apjaa4eefbib44L., Nyman, L. Å., \& Olofsson, H. 2003, A\&A, 402, 617

Zack, L. N., \& Ziurys, L. M. 2013, ApJ, 765, 112

Zeigler, N., Zack, L. N., Woolf, N. J., \& Ziurys, L. M. 2013, ApJ, 778, 16

Zhang, Y., Kwok, S., \& Trung, D.-V. 2008, ApJ, 678, 328

Zhang, Y., Kwok, S., Nakashima, J., Chau, W., \& Trung, D.-V. 2013, ApJ, 773,71

Ziurys, L. M., Adande, G. R., Edwards, J. L., et al. 2015, OLEB, 45, 275

Ziurys, L. M., Halfen, D. T., Geppert, W., \& Aikawa, Y. 2016, AsBio, 16, 997 